\title{
Nature-Inspired Optimization Algorithms
}




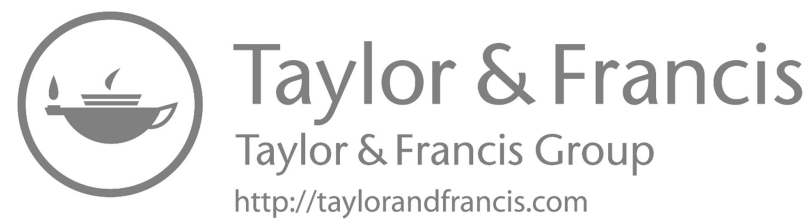




\section{Nature-Inspired Optimization Algorithms}

A Vasuki

CRC CRC Press

Taylor \& Francis Group Boca Raton London New York 
First edition published 2020

by CRC Press

6000 Broken Sound Parkway NW, Suite 300, Boca Raton, FL 33487-2742

and by CRC Press

2 Park Square, Milton Park, Abingdon, Oxon, OX14 4RN

(C) 2020 Taylor \& Francis Group, LLC

CRC Press is an imprint of Taylor \& Francis Group, LLC

Reasonable efforts have been made to publish reliable data and information, but the author and publisher cannot assume responsibility for the validity of all materials or the consequences of their use. The authors and publishers have attempted to trace the copyright holders of all material reproduced in this publication and apologize to copyright holders if permission to publish in this form has not been obtained. If any copyright material has not been acknowledged please write and let us know so we may rectify in any future reprint.

Except as permitted under U.S. Copyright Law, no part of this book may be reprinted, reproduced, transmitted, or utilized in any form by any electronic, mechanical, or other means, now known or hereafter invented, including photocopying, microfilming, and recording, or in any information storage or retrieval system, without written permission from the publishers.

For permission to photocopy or use material electronically from this work, access www.copyright.com or contact the Copyright Clearance Center, Inc. (CCC), 222 Rosewood Drive, Danvers, MA 01923, 978-750-8400. For works that are not available on CCC please contact mpkbookspermissions@tandf.co.uk

Trademark notice: Product or corporate names may be trademarks or registered trademarks, and are used only for identification and explanation without intent to infringe.

MATLAB $^{\circledR}$ and Simulink ${ }^{\circledR}$ are trademarks of the MathWorks, Inc. and are used with permission. The MathWorks does not warrant the accuracy of the text or exercises in this book. This book's use or discussion of MATLAB ${ }^{\circledR}$ and Simulink ${ }^{\circledR}$ software or related products does not constitute endorsement or sponsorship by the MathWorks of a particular pedagogical approach or particular use of the MATLAB ${ }^{\circledR}$ and Simulink ${ }^{\circledR}$ software. 


\section{Contents}

Preface

Author xiii

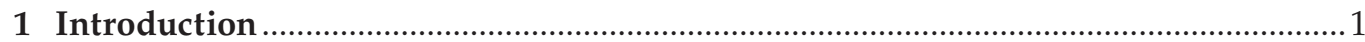

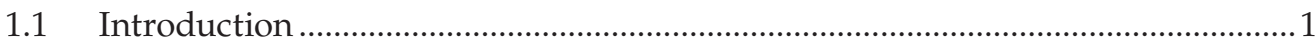

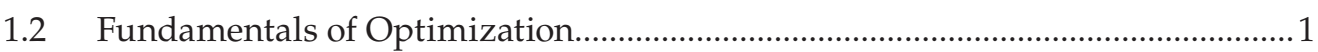

1.3 Types of Optimization Problems .......................................................................

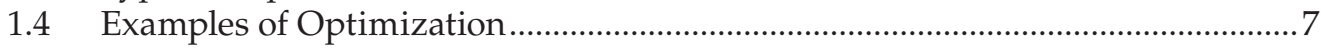

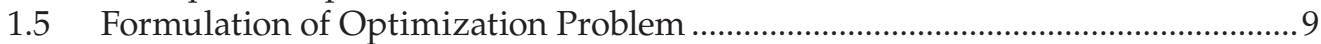

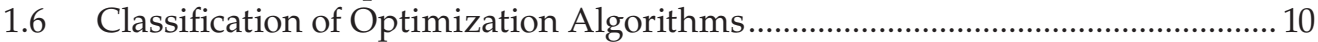

1.7 Traveling Salesman Problem and Knapsack Problem .......................................... 14

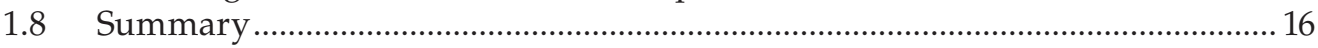

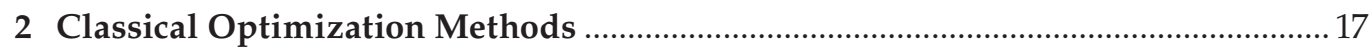

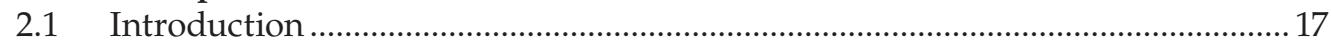

2.2 Mathematical Model of Optimization ............................................................ 18

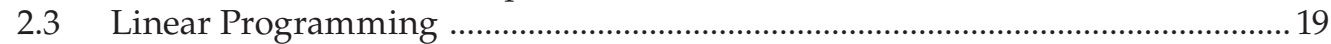

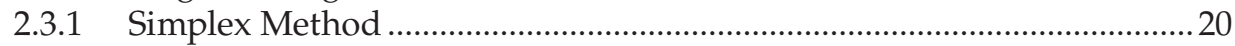

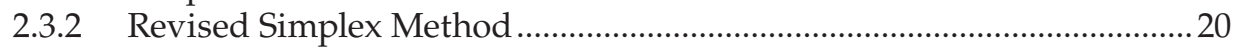

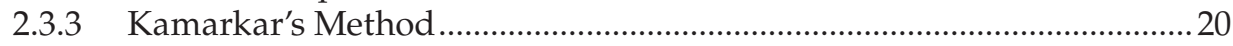

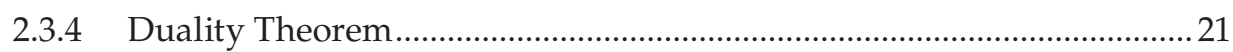

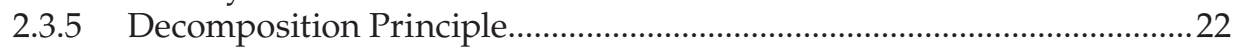

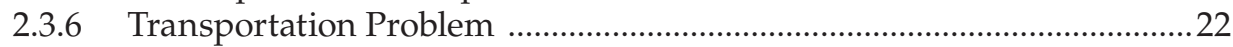

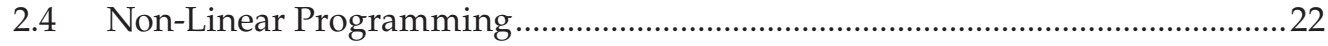

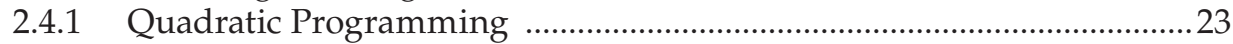

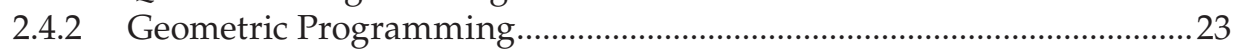

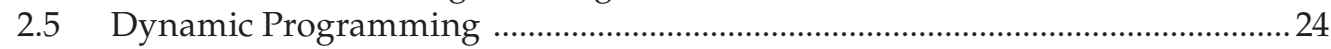

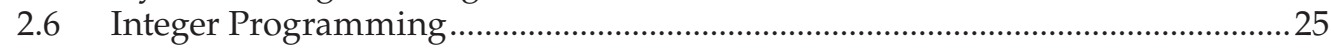

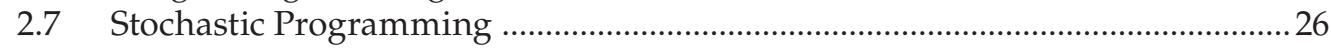

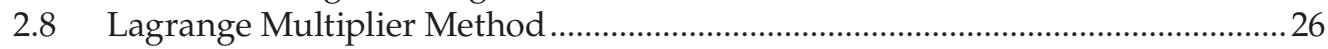

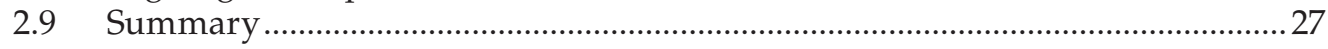

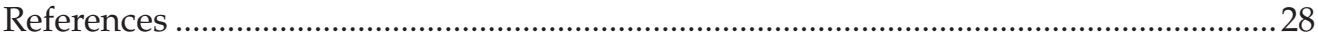

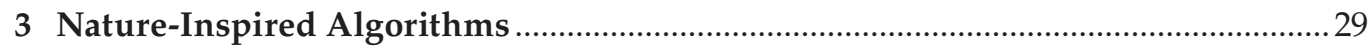

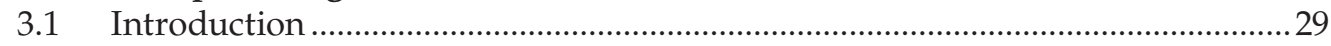

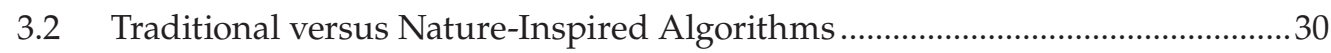

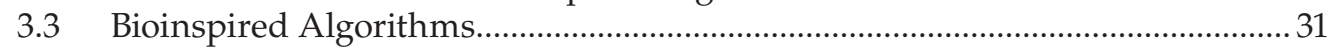

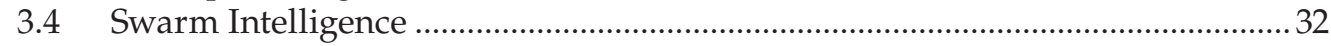

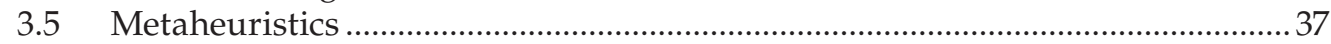

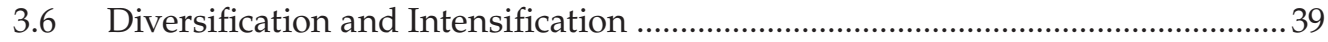

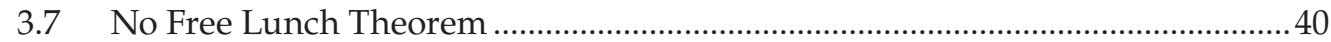

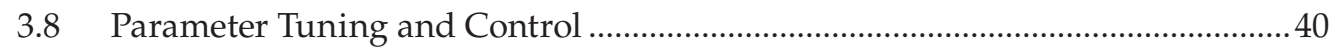

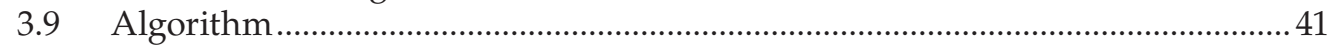




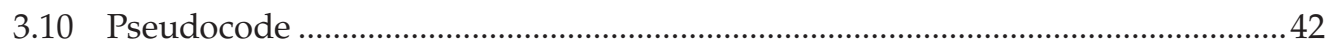

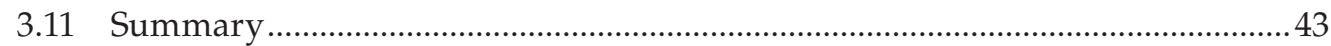

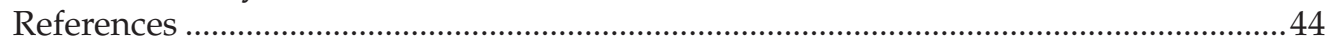

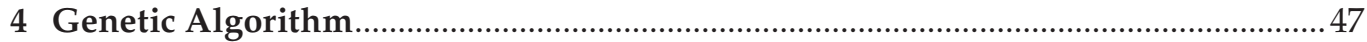

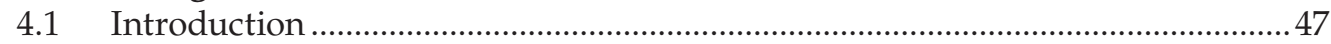

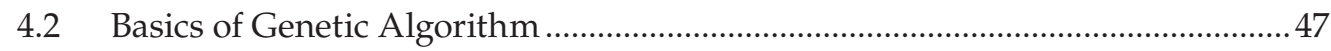

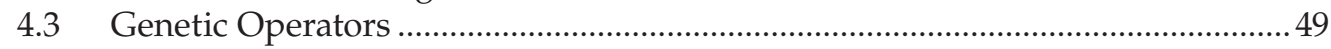

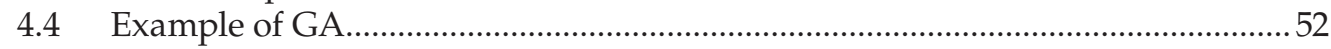

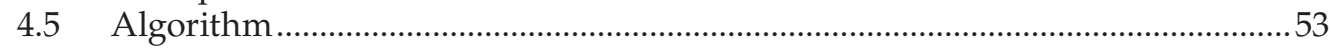

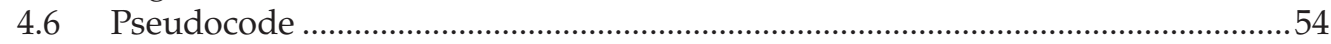

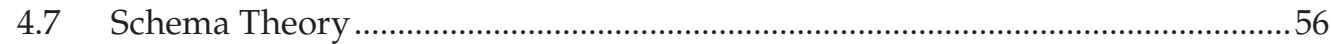

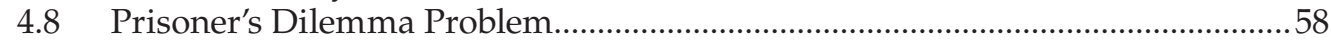

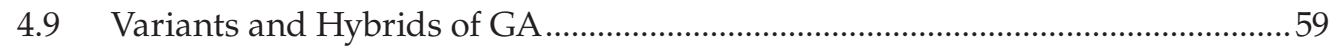

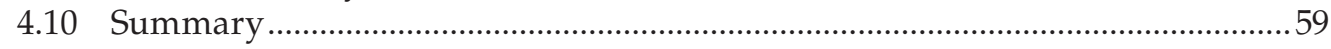

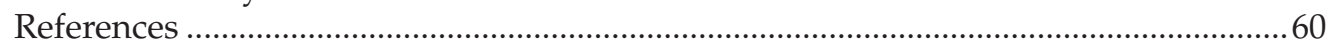

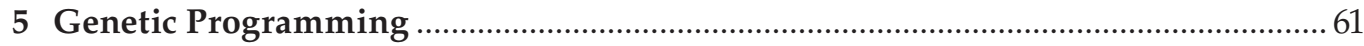

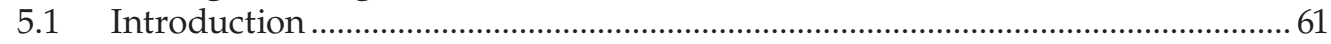

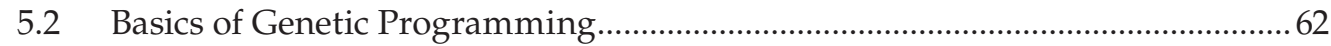

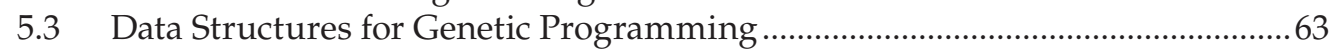

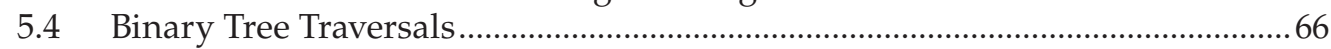

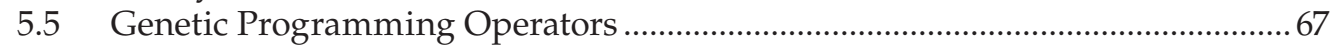

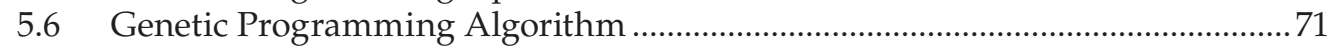

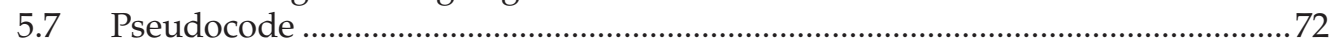

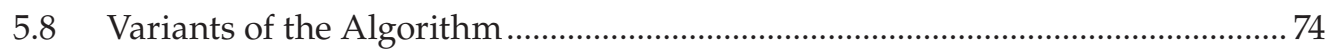

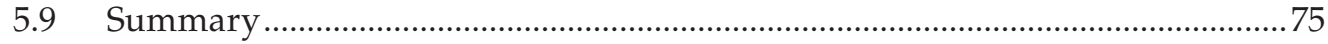

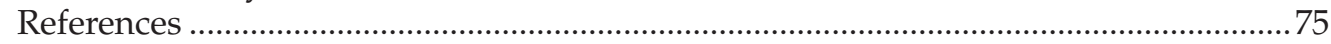

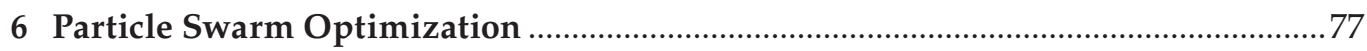

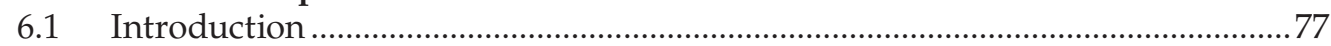

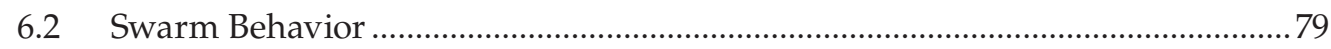

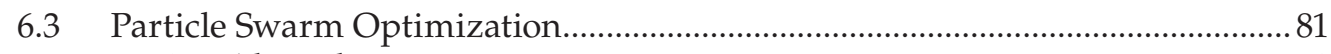

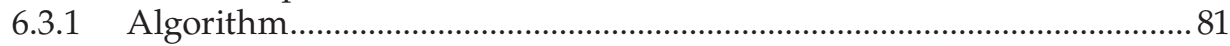

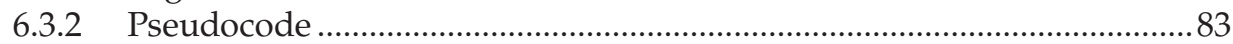

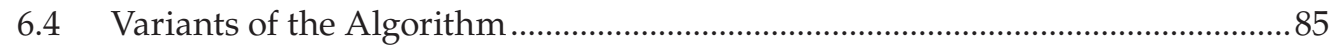

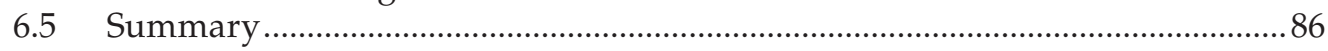

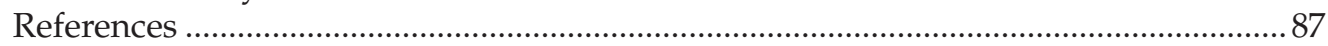

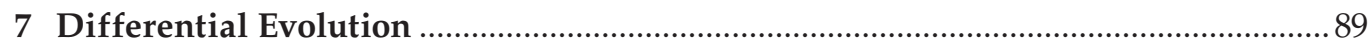

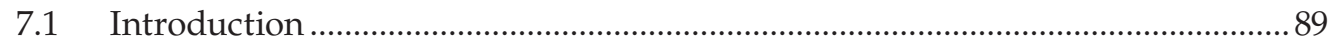

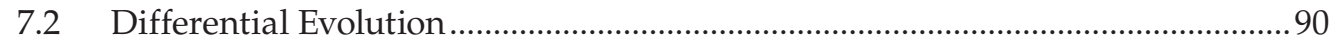

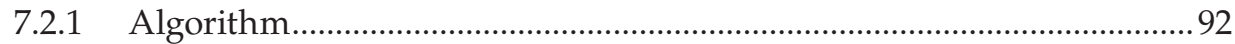

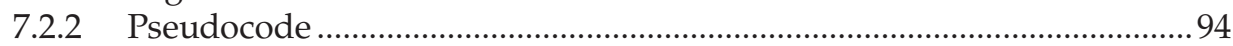

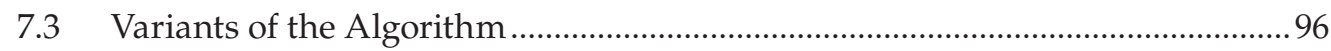

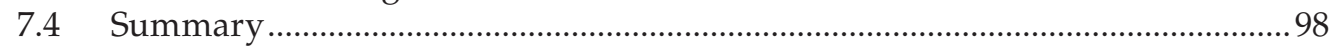

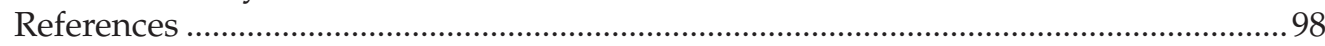




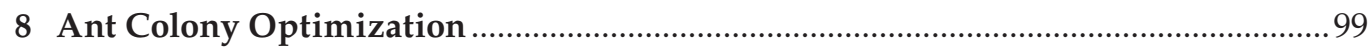

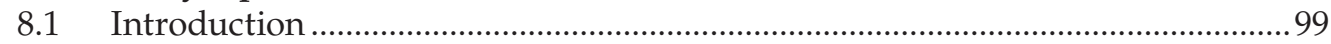

8.2 Ant Colony Characteristics .................................................................................99

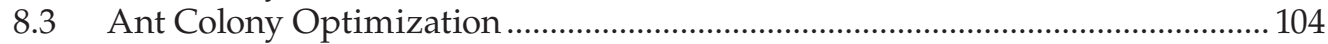

8.3.1 Traveling Salesman Problem ...……………………………………….... 105

8.3.2 Algorithm.......................................................................................... 106

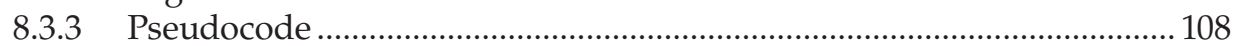

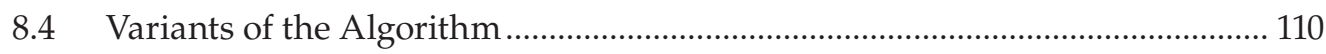

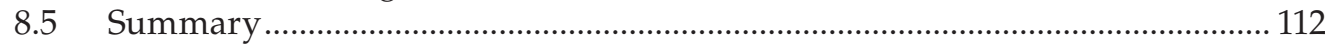

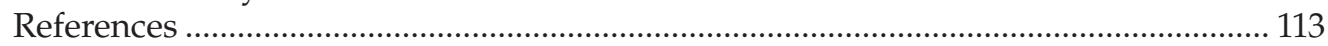

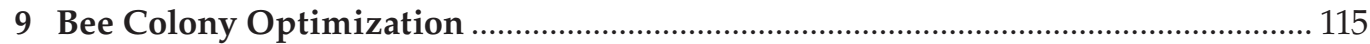

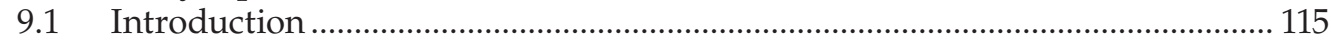

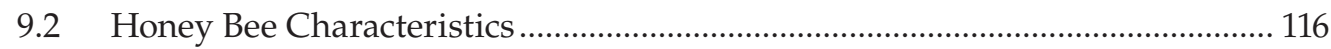

9.3 Bee Colony Optimization ………………………......................................... 121

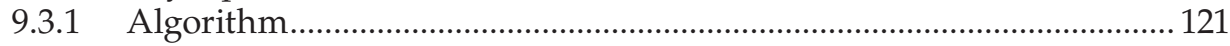

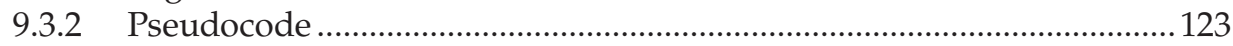

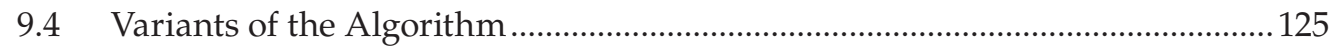

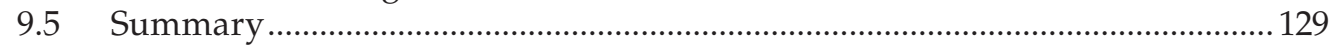

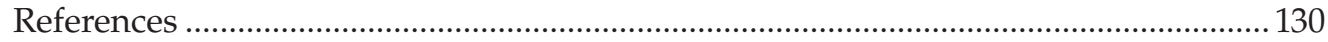

10 Fish School Search Algorithm ................................................................................ 131

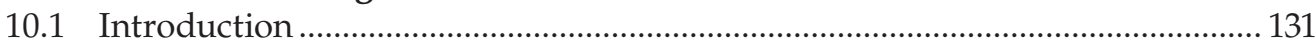

10.2 Fish School Behavior.............................................................................................. 131

10.3 Fish School Search Optimization ………………………………………….... 135

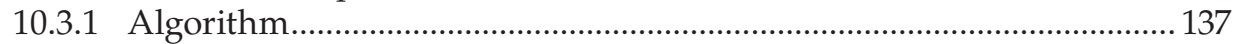

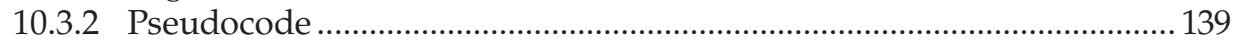

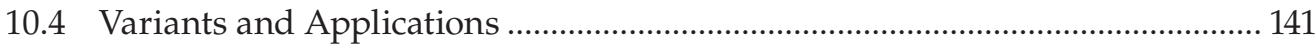

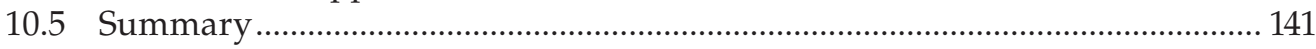

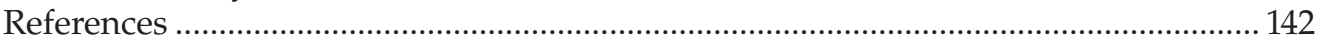

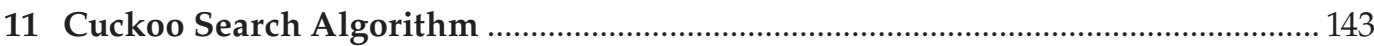

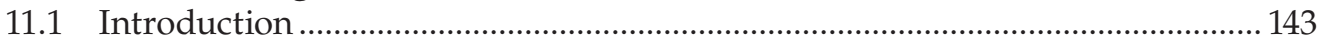

11.2 Cuckoo Bird Behavior..................................................................................... 143

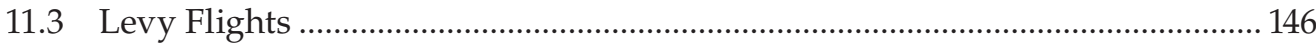

11.4 Cuckoo Search Optimization ............................................................................ 147

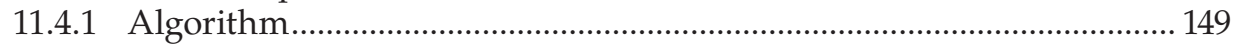

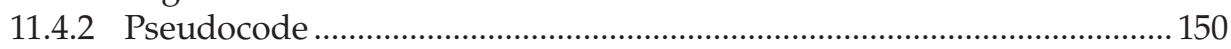

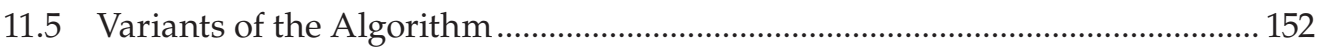

11.5.1 Discrete Cuckoo Search Algorithm.......................................................... 152

11.5.2 Binary Cuckoo Search (BCS) Algorithm .................................................. 152

11.5.3 Multi-Objective Cuckoo Search Algorithm (MOCS)................................. 153

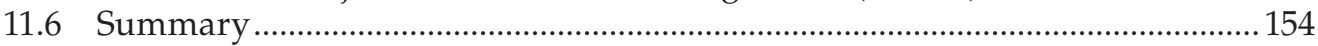

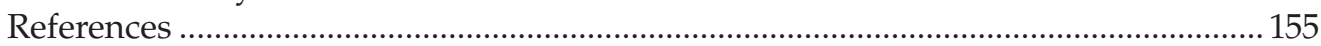

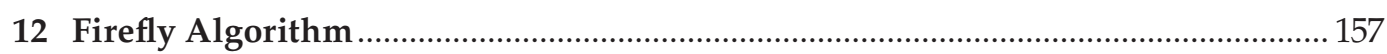

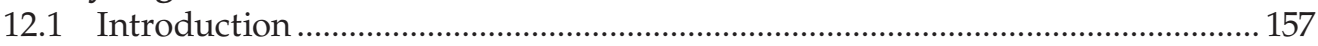

12.2 Firefly Behavior and Characteristics ................................................................... 157 
12.3 Firefly-Inspired Optimization .................................................................... 160

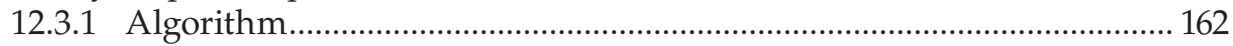

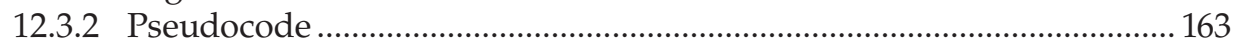

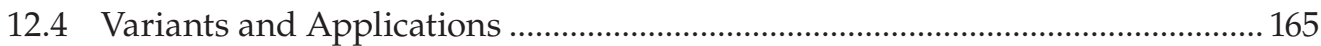

12.5 Summary

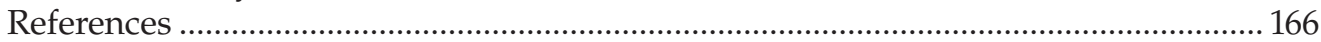

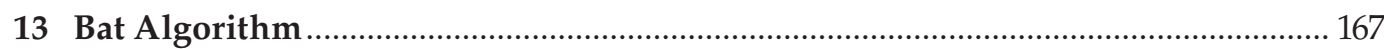

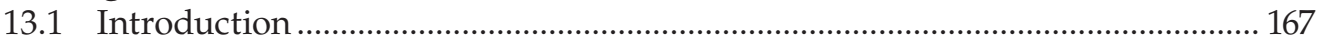

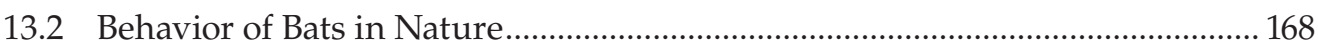

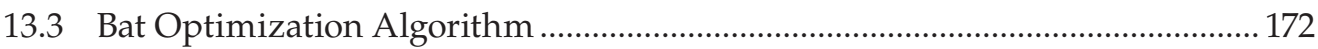

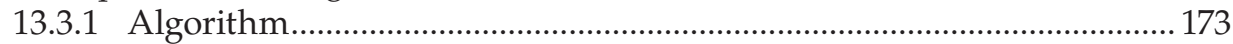

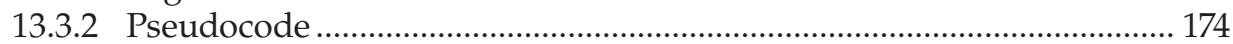

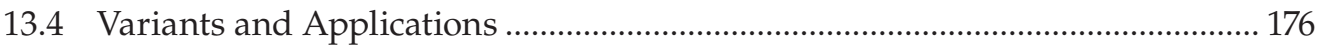

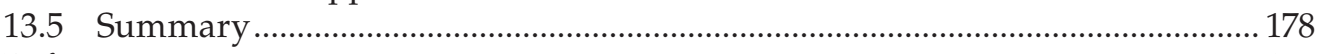

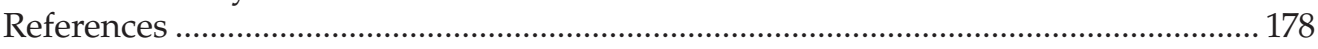

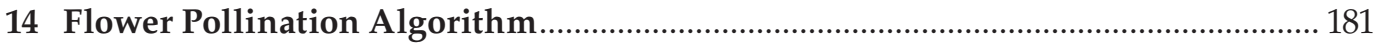

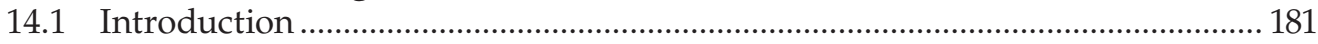

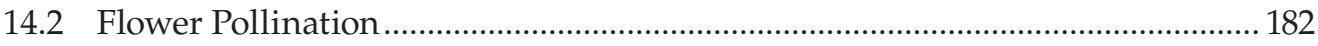

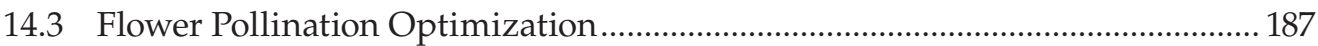

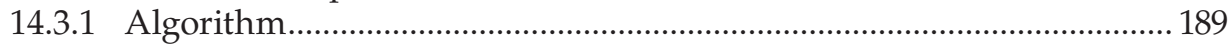

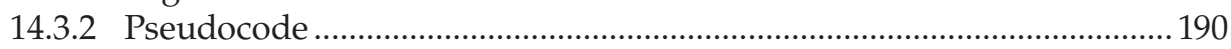

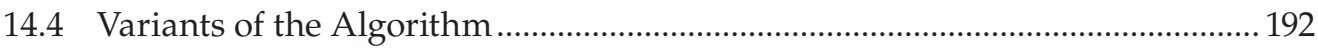

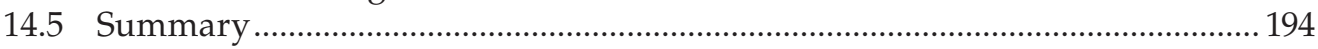

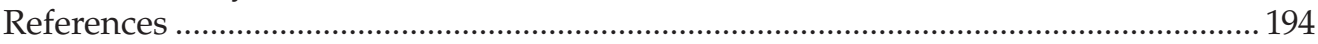

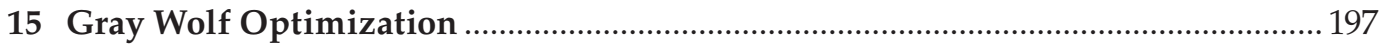

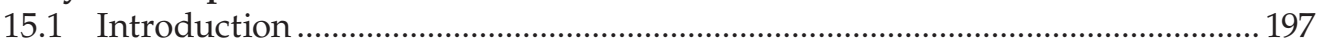

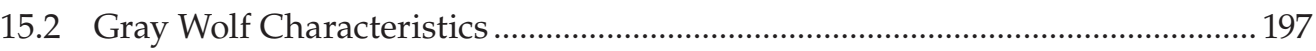

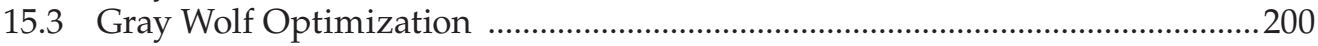

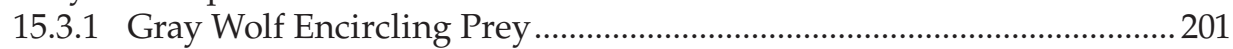

15.3.2 Hunting Behavior of Gray Wolves ...................................................... 202

15.3.3 Attacking of Prey by Gray Wolves ....................................................... 202

15.3.4 Gray Wolves Searching for Prey (Exploration)........................................203

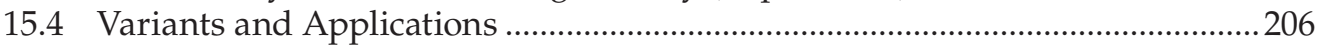

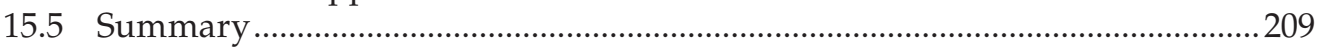

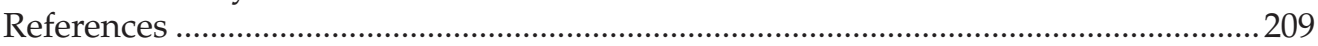

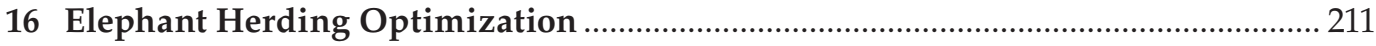

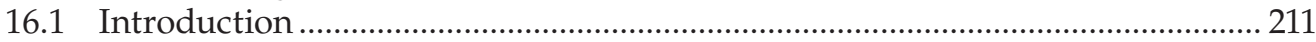

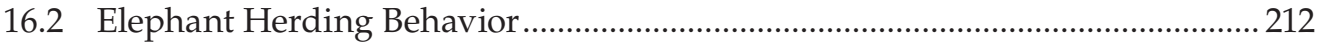

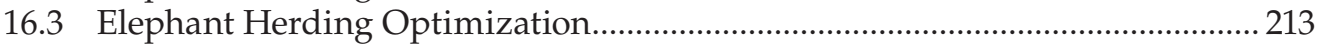

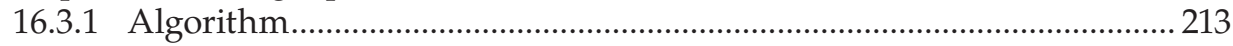

16.3.2 Pseudocode ......................................................................................... 215

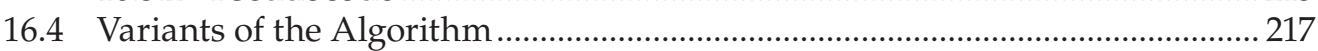

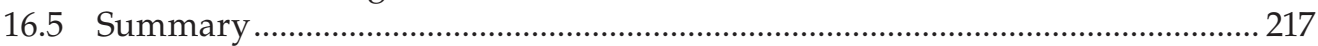

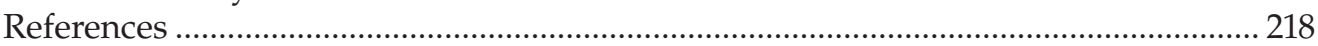




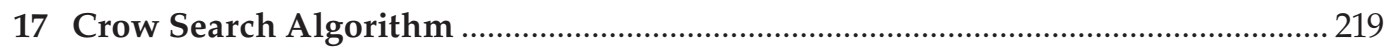

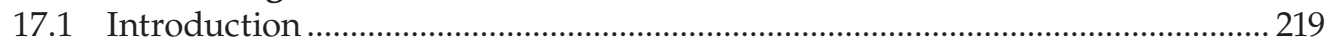

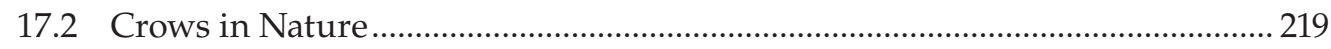

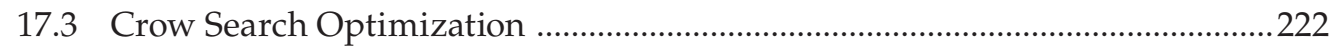

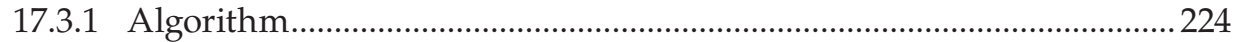

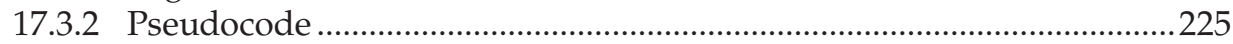

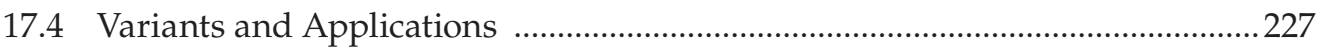

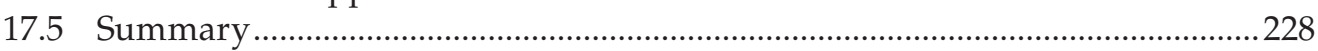

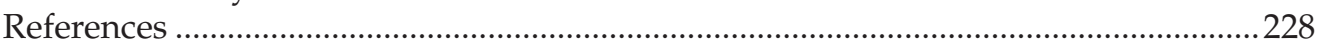

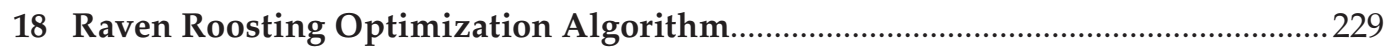

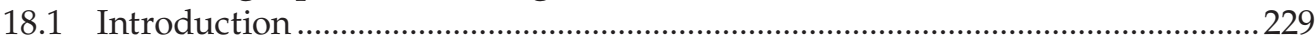

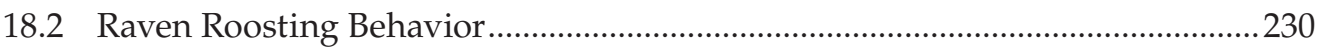

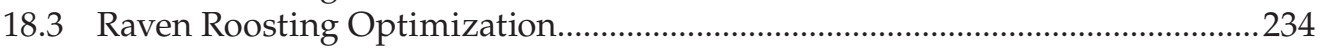

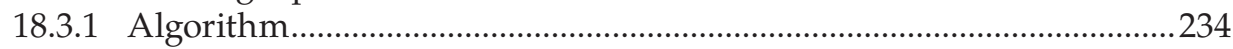

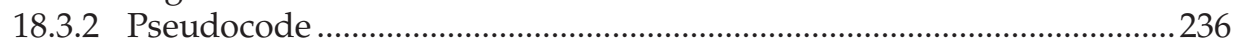

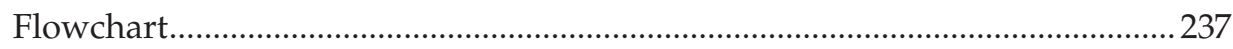

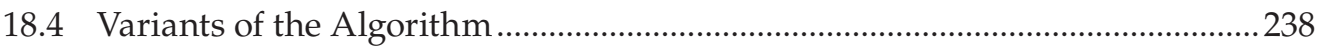

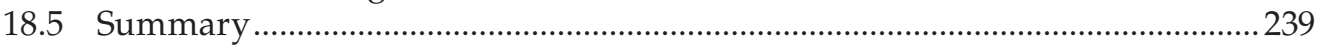

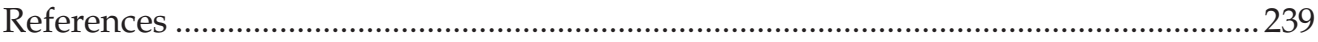

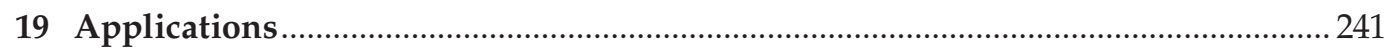

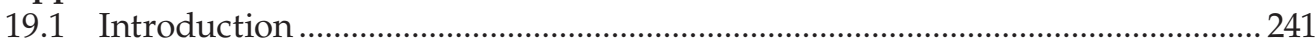

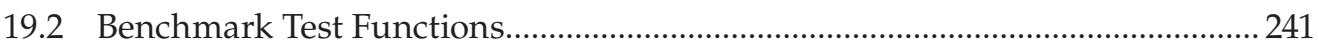

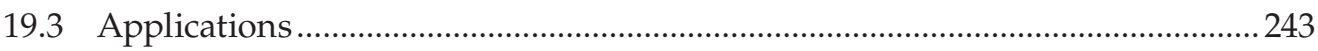

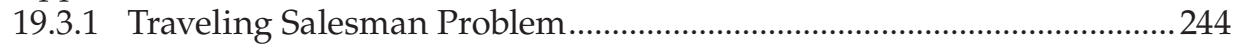

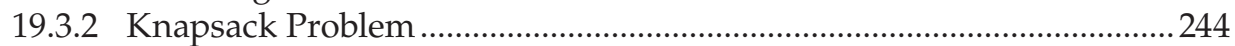

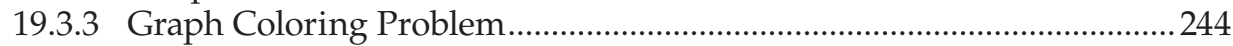

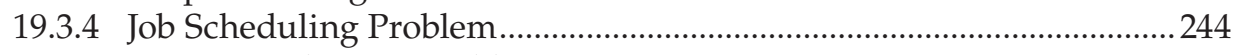

19.3.5 Feature Reduction Problem ..................................................................... 244

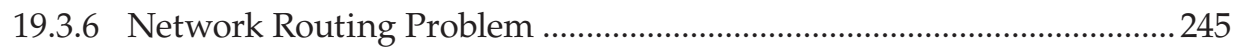

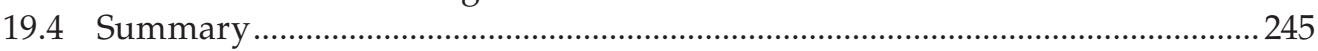

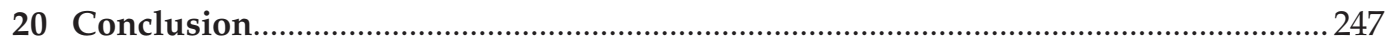

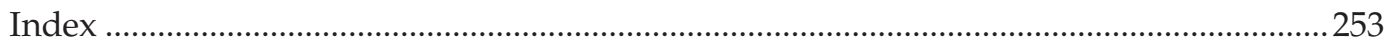




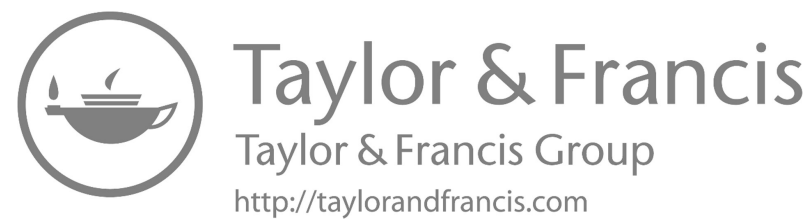




\section{Preface}

This book is intended as a reference for undergraduate and postgraduate students as well as researchers who are working on complex problems to find optimum solutions. The book can serve as a reference for faculty who are handling classes on the topic of optimization. It gives a general overview of optimization and its applications with simple examples and discusses conventional optimization techniques and their limitations. Fifteen natureinspired optimization algorithms have been included with a detailed explanation of the techniques.

These optimization algorithms can be applied to any real-life problem, either constrained or unconstrained. It involves choosing values of parameters associated with the problem in order to arrive at the optimum solution. The unconventional optimization algorithms are broadly based on biological evolution and physical and chemical processes. These nature-inspired algorithms have been developed from the study of swarm behavior of animals, birds, and insects. The main advantage is that they are population-based, and hence the search for the optimum solution can be conducted in parallel by multiple agents. This reduces the time taken to arrive at the (best) global optimum solution to the problem. Since the problems could be multimodal with several local optima, the algorithm should be able to distinguish between local and global optimum solutions.

These algorithms can be tested with standard benchmark data sets and classical engineering design problems. The standard problems in computer science such as the traveling salesman, graph coloring, finding the shortest path in a graph, job scheduling, and routing in computer networks are NP-hard. Image processing is another broad area that includes enhancement, segmentation, compression, classification, object recognition, feature selection, clustering, and registration. The application of nature-inspired algorithms to such intractable problems leads to optimum solutions in the shortest possible time. A list of comprehensive problems upon which these algorithms can be tested is also provided.

I sincerely thank everyone who have helped me in completing this book.

Dr. A Vasuki 


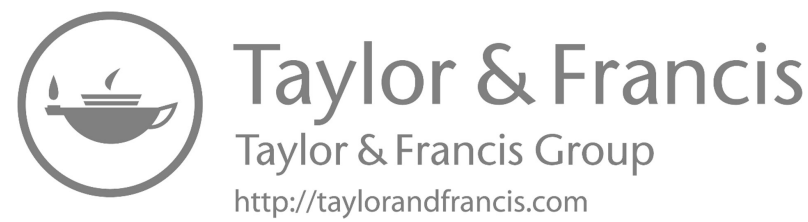




\section{Author}

Dr. A Vasuki is currently working as Professor in the Department of Mechatronics Engineering at Kumaraguru College of Technology, Coimbatore, India. She has 28 years of teaching, research and academic administration experience. She has completed B.E in Electronics and Communication Engineering from PSG College of Technology in 1989. She has completed her postgraduate degree M.E Applied Electronics from Coimbatore Institute of Technology in 1991. She has done her Ph.D in Image Compression from PSG College of Technology under Anna University Chennai in 2010. Her research interests are Signal Processing, Image Processing, Communication and Optimization. She has published 3 Book Chapters, 38 National and International Journal papers and 60 National and International Conference papers. She has guided 30 PG projects and 50 UG projects. She is an approved Research Supervisor under Anna University Chennai and is currently guiding 9 research scholars. 


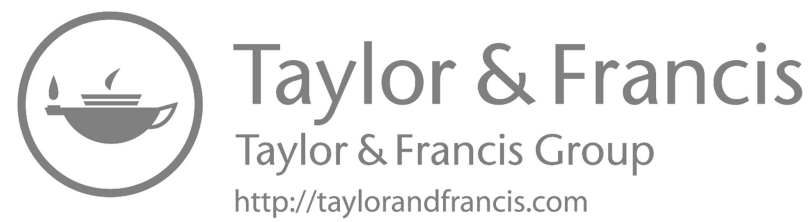




\section{Introduction}

\subsection{Introduction}

Optimization is of prime importance in most of our everyday activities and plays a vital role in achieving desired goals. Optimization is arriving at the best set of parameters, maybe by trial and error, so that we achieve the desired objective(s) exactly or at least a good approximation. We optimize parameters to achieve desired objectives without being consciously aware of it many times in our day-to-day activities. Optimization can be applied in diverse fields such as mathematics, physics, chemistry, engineering design, production, telecommunication, networks, computer science, economics, management, etc. The problems range from the very simple to the highly complex ones like designing steel structures, aircraft routes, road connectivity, routing in computer networks, unmanned space missions, autonomous vehicles, manufacturing, automation, robots, and so on. The goal of optimization could be to maximize quality/profit/efficiency or minimize cost/ wastage/resources utilized with reduced time, space, and computational complexity. The problems might have constraints that have to be satisfied in finding the optimal solution. The constraints could be limits on length, area, power consumed, cost, weight, or restriction on certain variables to assume integer values (positive or negative), and some variables need to be bounded between minimum and maximum values as specified in the problem. In all optimization problems there will be several parameters that have to be adjusted to get the best possible output. The problems to be solved are generally complex in nature with many conflicting requirements. The solution attained has to accommodate all these various conflicting requirements, and, keeping the objective(s) in focus, the optimization technique has to be designed.

\subsection{Fundamentals of Optimization}

Optimization is obtaining the optimum values of a set of variables upon which an objective function depends, and that could be either constrained or unconstrained. Any set of values assigned to the variables always produces an output but the optimum set of values produces the optimum output. The objectives of the problem and the constraints, if any, can be formulated in terms of mathematical functions or equations. The mathematical expression representing the goal of optimization is called the objective function. The objective function could be defined for maximization or minimization depending on the problem domain. Figure 1.1 illustrates the general optimization problem with input 


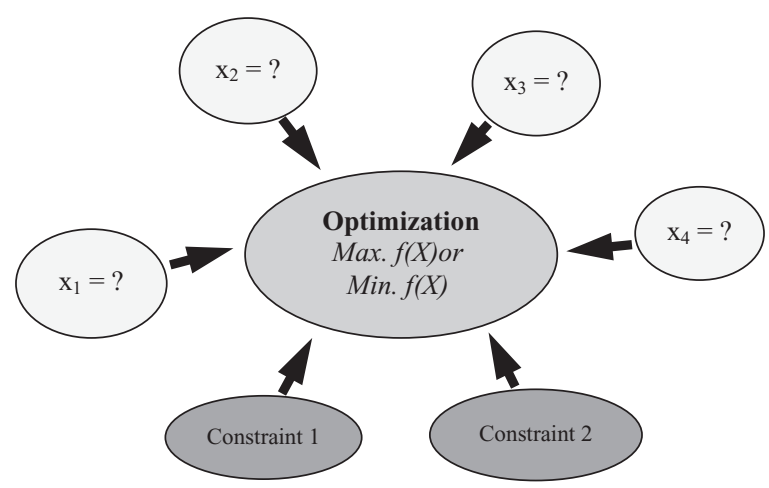

FIGURE 1.1

General optimization problem.

variables and constraints that either maximizes or minimizes the objective function. In Figure 1.1 the objective function $f(X)$ depends on the vector of input variables represented by $X=\left[\begin{array}{llll}x_{1} & x_{2} & x_{3} & x_{4}\end{array}\right]$.

When an objective function is to be maximized, it is called a fitness or quality function. When it is to be minimized, it is called a cost or penalty function. For a minimization function, if the minimum value is zero it is called an error function. The sign of the objective function can be complemented (+/-) in order to transform a maximization problem to minimization and vice versa. An objective function that is to be maximized can also be minimized by taking the negative of the function.

Some of the characteristics of the objective function are:

- The number of variables in the function is the dimension $(d)$ of the search space.

- Whether the variables assume continuous or discrete values determines whether the function is continuous or discrete.

- If the function is continuous, is it differentiable at all points in the search space?

- Whether the function has one maxima (unimodal) or multiple maxima (multimodal).

- Is the function unconstrained or constrained? If constrained, how many and what are the constraints? Are they equality or inequality constraints?

Mathematically, a function is represented as $f: R^{d} \rightarrow R$, where the function $f$ belongs to the $d$-dimensional hyperspace $R^{d}$. The domain $R^{d}$ is the parameter or search space with each $X \in R^{d}$ being a possible candidate solution to the objective function $f(X)$. The function $f(X)$ maps the search space to the function space $R$. The problem is to find $X^{*} \in R^{d}$, for which $f\left(X^{*}\right) \geq f(X), \forall X \in R^{d}$. This applies to a maximization problem, and for minimization problems, it has to be $f\left(X^{*}\right) \leq f(X), \forall X \in R^{d}$. The search space could be either the entire $d$-dimensional hyperspace or a subset of the hyperspace. When there are constraints associated with the problem, the search space becomes a subset of the $d$-dimensional hyperspace. The characteristics associated with the constraints of the problem are:

- Number of constraints for the problem

- Whether the constraints are equality or inequality constraints 


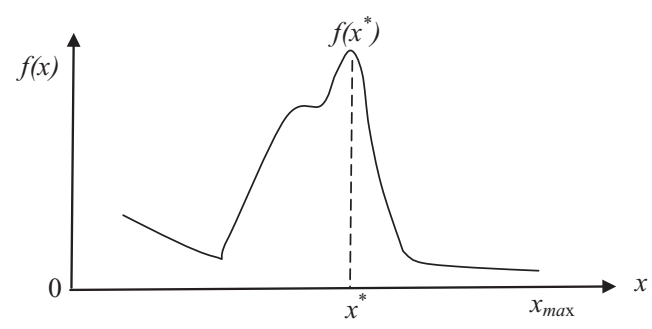

FIGURE 1.2

One-dimensional function $f(X)$ where $X=[x]$.

Figure 1.2 shows an objective function $f(X)$ where the vector $X=[x]$ is one-dimensional, since the function depends on only one variable $x$.

The one-dimensional plot of the objective function $f(X)$ shown in the Figure 1.2 has a maximum value at $x=x^{*}$. This is the global optimum since this is the highest value for the function $f(X)$. The search space is the one-dimensional $x$-axis where the continuous variable $x$ ranges from zero to a maximum value $x_{\max }$. In general, the search space is $S \rightarrow R^{d}$. The convergence of the algorithm or the final value attained is the optimum solution and depends to a great extent on the starting point in the search space or the initial value of $x$. If the negative of the function $f(X)$ is taken, the maximum value becomes the minimum value or the peak becomes a valley. The maximization problem becomes a minimization problem when the global minimum occurs at $X=x^{*}$.

Extending this concept further, the one-dimensional function $f(X)$ could have multiple maxima or minima, as illustrated in Figure 1.3. The function $f(X)$ shown in Figure 1.3 has multiple peaks and valleys, where $X=[x]$. The peak with the highest value is called the global maximum and the valley with the lowest value is called the global minimum. The other peaks which are smaller than the maximum are called local maxima, and the valleys which have higher values than the minimum are called local minima. The search for the optimum solution for $f(X)$ starts somewhere along the curve and the objective is to find the global maximum or minimum, as the case may be. The problem occurs when the search for the optimum solution gets trapped in local maxima or minima. The optimization algorithm should be such that the function should attain the global optimum value and if it gets trapped in local optimum it should be able to come out of the local optimum point or region in the search space.

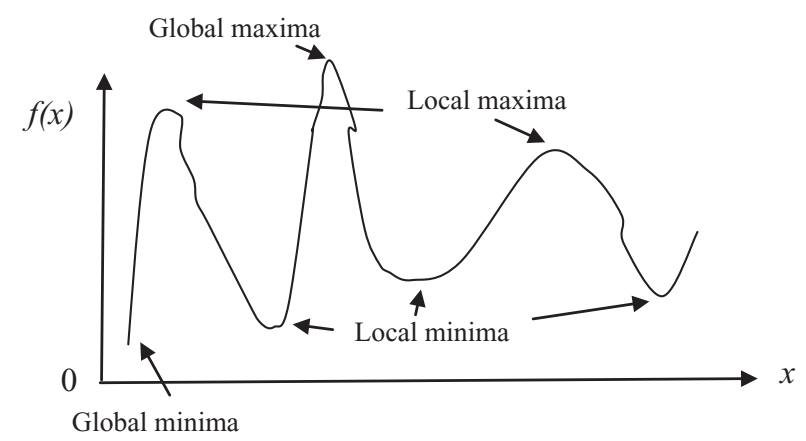

FIGURE 1.3

One-dimensional function $f(X)$ having multiple maxima and minima. 
In the above problem the function $f(X)$ is dependent on a single variable $x$; hence it is single variable optimization. This maximization problem of one variable can be extended to $d$ number of variables, thus making the objective function $d$-dimensional and the search space becomes a $d$-dimensional hyperspace. In effect, this becomes multivariable optimization. As another example, consider a function of two variables $f(X)=f\left(x_{1}, x_{2}\right)$ with only one global minima. The two-dimensional search space of a function of two variables $f(X)$ $=f\left(x_{1}, x_{2}\right)$ is shown as a contour plot in Figure 1.4. The global minima appears nearly at the center of the search space, as indicated.

In general,

$$
f(X)=f\left(x_{1}, x_{2}, \ldots x_{d}\right)
$$

and the search space is $\rightarrow R^{d}$. This is single-objective optimization problem since there is only one objective function that is to be maximized or minimized. In contrast to this, if there is more than one objective function to be maximized or minimized then it becomes multi-objective optimization. If there are no constraints attached to the problem it is unconstrained optimization whereas if there are one or more constraints in the problem it is constrained optimization. The constraints of the problem could be equality or inequality constraints. They are mathematically represented as:

$$
\begin{array}{r}
g_{i}(X)=0, \quad i=1,2, \ldots . P \\
h_{j}(X) \geq 0, \quad j=1,2, \ldots . Q \\
\text { or } h_{j}(X) \leq 0, \quad j=1,2, \ldots . Q
\end{array}
$$

where the number of equality constraints is $P$ and the number of inequality constraints is $Q$. When there are multiple number of objective functions, the problem is multi-objective optimization. This is mathematically represented as:

$$
f\left(X_{k}\right)=f\left(x_{k 1}, x_{k 2}, \ldots \ldots, x_{k d}\right), \quad k=1,2, \ldots . . K
$$

where $K$ is the number of objective functions that are to be either maximized or minimized. The objective function $f(X)$ maps the search space to the function space, which will

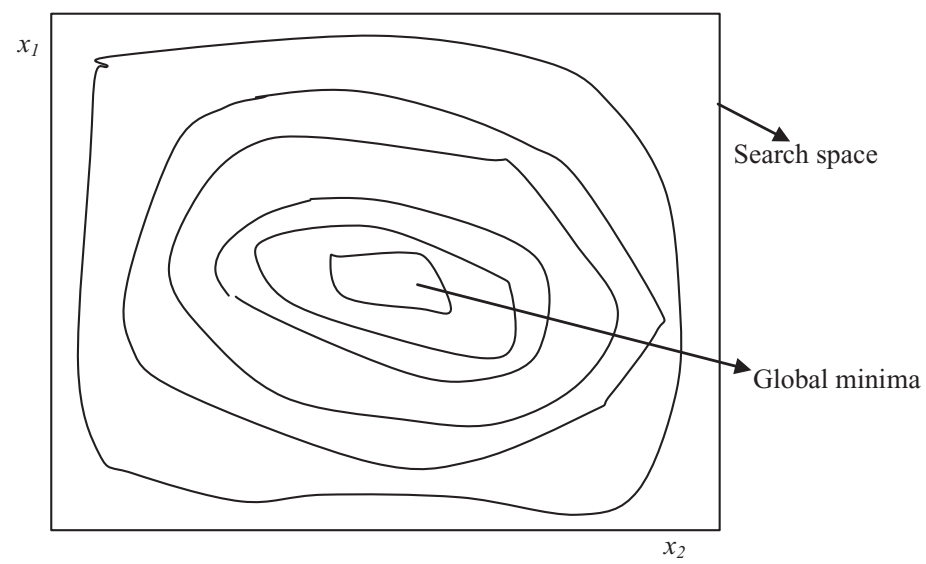

FIGURE 1.4

Two-dimensional search space of a function $f\left(x_{1}, x_{2}\right)$ with one global minimum. 
have a single value for single-objective optimization and multiple values in the case of multi-objective optimization. The parameters or variables can be continuous or discrete and belong to a finite or infinite set. The parameters could be interdependent or they may be independent of each other. The function might have only one maximum point that is the global maximum called a unimodal function, or it could have one global maximum and multiple local maxima called a multimodal function. The example plotted in Figure 1.2 is unimodal whereas the plot shown in Figure 1.3 is multimodal.

\subsection{Types of Optimization Problems}

Optimization problems usually start with the definition of the problem such as minimization of cost, energy, resources used, or maximization of profit and quality. From this problem statement the objective function is formulated, either as a maximization or minimization function. The next step is to identify the constraints associated with the problem, which could be either equality or inequality constraints. The parameters associated with the objective function and the constraints have to be identified and their boundaries clearly stated. The optimum solution for the problem has to be found by searching, for which the search or solution space needs to be defined. The tentative location of the solution in the search space or the local region where the solution could possibly be found also has to be initially known, because that is the point at which the search for the optimum has to begin. If the information about the local region where the solution is likely to be found is not available, then the search for the optimum has to start from a random location in the search space. If the space is quite large and multidimensional, it is neither practical nor feasible to do an exhaustive search of the solution space. If there are no objectives in the problem but only constraints, then it is a feasibility problem. If the objective function and constraints are separable in the design variables, it is a separable optimization problem. Given any mathematical function of design variables and constraints, if it is possible to separate them in terms of the variables then it is a separable problem. Let a function of three design variables be defined as $f(X)=x_{1} x_{2} x_{3}$. Taking logarithm on both sides, $\log [f(X)]=$ $\log \left(x_{1} x_{2} x_{3}\right)=\log x_{1}+\log x_{2}+\log x_{3}$. Since this is separable in terms of the three variables $x_{1}$, $x_{2}$, and $x_{3}$, it is a separable problem.

The optimization problems and techniques are categorized into several classes based on the characteristics of the objective function, the associated variables, and constraints.

Continuous and Discrete Optimization: When the objective function is a continuous function of design variables it is continuous optimization. This continuous function could be differentiable or non-differentiable. If the function is differentiable, the traditional or classical methods of optimization can be applied. $f(x)=12 x^{3}+5 x^{2}-2 x+4$ is an example of a continuous function that is differentiable, and the traditional methods of optimization can be applied to find the minima or maxima of the function $f(x)$. If the function is nondifferentiable, then other methods have to be explored, or they have to be transformed such that the classical methods can be applied. Such non-differentiable functions are usually not smooth and have sharp discontinuities where the derivatives do not exist. When the objective function is dependent on a set of independent discrete variables it is discrete or combinatorial optimization. Sometimes, the function could be a mix of continuous and discrete variables and it becomes a mixed optimization problem. An example of a combinatorial optimization problem is finding the minimum spanning tree of a connected 


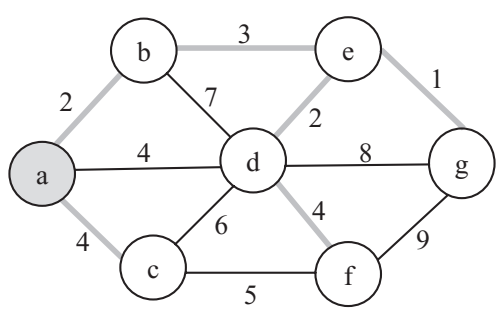

\section{FIGURE 1.5}

Minimum spanning tree.

undirected graph. The problem is to connect all the nodes of the graph such that the total weight of the edges connecting these nodes is minimum. In addition, there should not be any cycles in the minimum spanning tree. Kruskal's and Prim's algorithms are two greedy algorithms that are normally applied in finding the minimum spanning tree of a graph.

Figure 1.5 shows an example of a connected graph, and its minimum spanning tree is shown highlighted. Initially starting from node $a$ of the graph, all the edges connecting this node are examined and the edge with the least weight of 2 is chosen and the node $b$ connecting this edge is included in the tree. The edges connected to nodes $a$ and $b$ that are not yet included in the tree are examined, and the edge with a weight of 3 connecting node $b$ to node $e$ is included in the tree. This process is repeated until all the nodes in the graph have been covered. The algorithm for obtaining the minimum spanning tree is a greedy algorithm since it chooses the edge with the smallest weight at each step. The final optimized output is the set of edges whose total weight is minimum with all the nodes of the graph being connected and included in the tree. This is an optimization problem with the objective function being the total weight of all the edges in the minimum spanning tree and the constraints being that each node be included only once and no cycles are formed.

Deterministic and Stochastic Optimization: Deterministic optimization algorithms are consistent, and each time the algorithm is run, it produces the same result for the same input values. Stochastic optimization algorithms have some randomness associated with them and might produce different results for each run of the algorithm. An example for the deterministic optimization algorithm is hill climbing and other traditional gradient-based methods such as the simplex method. Genetic algorithm and particle swarm optimization are two famous examples of population-based stochastic optimization techniques. Stochastic optimization algorithms are heuristic or metaheuristic and have some inherent randomness that leads to the optimum or near-optimum solution in finite time.

Constrained and Unconstrained Optimization: When there are no constraints on the problem to be solved it is unconstrained optimization. When there are constraints related to the problem, it is constrained optimization. Let $f\left(x_{1}, x_{2}\right)=x_{1}{ }^{2}-10 x_{1}+5 x_{2}^{2}-2 x_{2}+3$ be the function to be optimized. The problem is to find the values of $x_{1}$ and $x_{2}$ that will maximize $f\left(x_{1}, x_{2}\right)$. If there is no restriction on the values that $x_{1}$ and $x_{2}$ can assume, it is unconstrained optimization. If it is stated that $x_{1}$ can only be a positive integer then it is constrained optimization. The constraints could be equality or inequality constraints. The constraint $x_{1} \geq 0$ is an inequality constraint whereas $2 x_{1}+x_{2}=6$ is an equality constraint. Constrained optimization problems are more difficult to solve than unconstrained optimization problems.

Linear and Non-linear Optimization: If the objective function to be optimized is linear then it is linear optimization. If the objective function is non-linear then it is non-linear optimization. An example for a linear objective function is $f(X)=2 x_{1}+4 x_{2}-7$, and a non-linear objective function is $f(X)=x_{1}^{2}+3 x_{1}-2 x_{2}^{2}+5$. Non-linear optimization problems are more 
difficult to solve than linear optimization problems. Similar to objective functions, the constraints also could be linear or non-linear, for example, $x_{1}+x_{2}=6$ is a linear constraint and $x_{1}^{2}+x_{2}^{2}>100$ is a non-linear constraint.

Single- and Multi-Objective Optimization: When there is only one objective function $f(X)$ to be optimized it is single-objective optimization. When there are multiple objective functions $\left\{f\left(X_{1}\right), f\left(X_{2}\right), \ldots, f\left(X_{K}\right)\right\}$ to be optimized it is multi-objective optimization. In multiobjective optimization there are multiple $(K)$ objective functions and each one is a function of $d$ number of design variables. In multi-objective optimization, there could be multiple functions with conflicting requirements. There will be no single design vector that will maximize (or minimize) all the objective functions. It might not be possible to satisfy all the constraints to get an optimum value for all the objective functions. It might be necessary to have tradeoffs when there are conflicting requirements between multiple objectives. A typical example with multiple objectives is manufacturing a car. One objective will be minimization of cost, the second will be minimization of fuel consumption, the third will be maximization of efficiency, the fourth will be minimization of resources used in manufacturing, the fifth will be minimization of emission of pollutants, and so on. Algorithms that work for single-objective optimization might not work directly for multi-objective optimization. One method to solve such multi-objective optimization problems is to construct a single-objective function using a weighted combination of the multiple objective functions such as:

$$
f(X)=c_{1} f\left(X_{1}\right)+c_{2} f\left(X_{2}\right)+\ldots+c_{K} f\left(X_{K}\right)
$$

where $c_{1}, c_{2}, \ldots, c_{K}$ are the weighting coefficients.

A better method to take care of these conflicting requirements is that the optimal values of all the objective functions lie on the Pareto Optimal Front. The Pareto Optimal Front theory states that it is not possible to get the maximum value for all the multiple conflicting objectives of a problem simultaneously. What is stated here for a maximization problem applies equally well for a minimization problem. The optimal method to solve this multi-objective problem with conflicting requirements is to ensure that the solutions to the multiple objective functions lie on the Pareto Optimal Front. When the fitness value of one objective function is increased (improved), the fitness value of another objective function will be decreased (degraded); that means it is not possible to get the maximum value for all the objective functions simultaneously with one solution vector. If a solution vector $X_{i}$ lies on the Pareto Optimal Front, it yields a vector of fitness values for all the objective functions of the problem. Comparing these fitness values, it occurs that if this vector $X_{i}$ yields maximum fitness for objective function $f\left(X_{i}\right)$ it might not be the case for the function $f\left(X_{j}\right)$. Another solution vector $X_{r}$ which is also on the Pareto Optimal Front will produce the maximum value for the objective function $f\left(X_{r}\right)$ but not for $f\left(X_{i}\right)$ or $f\left(X_{j}\right)$. Therefore, any solution vector $X$ for a multi-objective optimization problem should lie on the Pareto Optimal Front for the solutions to be non-dominated or Pareto Optimal.

\subsection{Examples of Optimization}

These algorithms have one thing in common, that is, they all strive to produce the best solution for a given problem within a limited set of resources. The problem can be a complex 
engineering design such as a CNC Machine, a process plant like paper manufacturing, pattern recognition, image classification, or an NP-hard problem (non-deterministic polynomial time) in computer science like the traveling salesman problem (TSP), and the limitations on the resources are the constraints within which the best solution for the problem is to be attained. It can be expressed in terms of mathematical equations that could be solved using different methods suitable for the problem. One of the most important evaluation criteria for these algorithms is the time, space, and computational complexity incurred in arriving at the optimum solution.

\section{Paper Manufacturing}

In a process plant like paper manufacturing, many parameters will be involved in producing the paper. The objective function could be a mathematical function that has to be maximized and indicates the quality of the paper produced. There are several processes involved in paper manufacturing such as pulp extraction, the right blend (proportion) of chemical additives, fixing temperature and/or pressure, drying, bleaching, etc. Choosing the appropriate values for the parameters involved in the various processes plays a crucial role in the color, thickness, and quality of the paper produced. This is an optimization problem where the parameters of the paper manufacturing process are equivalent to the decision variables $X=\left\{x_{1}, x_{2}, \ldots, x_{d}\right\}$ in the search space and the function $f(X)$ is the fitness or quality function. The value of the fitness function has to be proportional to the paper quality produced. The global optimum of the objective function will be attained when the function has the highest value and the paper produced is of the best quality.

\section{Pattern Recognition}

Consider the pattern recognition problem where the objective is to identify and classify objects in an image with 100\% accuracy. Every object such as jasmine, rose, car, lorry, bike, building, human face, and fingerprint present in an image has its own set of features that enables recognition and classification. Flowers have features like the number of petals, length and width of the petals, color, diameter of the flower, etc. Similarly a fingerprint has features like ridges and minutiae. The ridge and minutiae patterns on the fingerprint enables classification and recognition.

\section{Feature Reduction}

Each object or pattern has its own unique set of features that enables identification. When the number of features of an object is higher it becomes impractical to extract all the features, compare it with the existing template, and then identify the object. So to circumvent the problem, a technique known as feature reduction is applied. This is the technique of selecting a minimum number of features from the entire set, for classification of objects with a reasonable percentage of accuracy. Minimizing the number of features is nothing but the selection of a subset of features, thus reducing the computational complexity in searching for the optimum solution. It is equivalent to minimizing the value of dimension $d$ in the hyperspace. Once $d$ is fixed, the next step is to find the value of the feature set $X$ $=\left\{x_{1}, x_{2}, x_{3}, \ldots, x_{d}\right\}$ so that $f(X)$ attains the global maximum or minimum value, as the case may be. If $f(X)$ represents classification accuracy then it will be maximized whereas if $f(X)$ is a classification error then it has to be minimized. 


\subsection{Formulation of Optimization Problem}

The first step in any problem solving is definition of the problem statement. Once the statement is clear, the objectives and constraints have to be outlined. The objective(s) of the problem have to be mathematically formulated as an objective function that is to be either maximized or minimized. The next step is to identify the limitations or boundaries of the design variables as equality or inequality constraints and write mathematical equations for them. When the equations are linear and differentiable, with few parameters, the standard classical methods can be applied. When the equations are non-linear and complex or if the objective function and/or constraints have discontinuities and are not differentiable, the classical methods could become intractable, necessitating the use of evolutionary or metaheuristic algorithms. The nature-inspired algorithms that are basically metaheuristic, population-based search algorithms will be a better choice for such complex problems. Many of the heuristic methods use a greedy criterion in accepting a new solution component that might evolve from the previous solution(s), if it is either going to increase the fitness value or decrease the cost. There is also a possibility of converging at a local optimum for such greedy algorithms, which could be overcome by the metaheuristic algorithms that search in parallel and have good diversity properties.

Although several mathematical techniques exist for solving optimization problems, the computational complexity involved might be too high in some of the cases. Certain approximations might reduce the search and computational complexity and help in arriving at the near-optimum solution in finite time for practical applications. When the number of design variables upon which the objective function is dependent is large, the dimensionality of the problem increases. This can be reduced by dimensionality-reduction methods based on the specific optimization problem. Sensitivity analysis of the objective function with respect to the parameters, i.e. change in the objective function value with changes in the design parameters could be done to improve the parameter settings and hence the performance of the algorithm. As another alternative, multilevel optimization could be applied for large complex problems. When the number of design variables and/or constraints becomes large, the optimization problem becomes unmanageable or impractical. Multilevel optimization involves breaking down an optimization problem of large size or dimensions into smaller sub-problems that can be optimized easily. The smaller sub-problems are linked to put together a solution for the larger problem. Yet another approach is parallel processing, where the sub-problems can be run independently in multiple parallel computers to speed up the optimization process.

The problem is to be defined clearly and the physical principles governing the system to be optimized are to be understood thoroughly. The limitations under which the solution is to be found are formulated as constraints. For example, the number of persons cannot be a fractional number, the resistance value cannot be negative, and so on. These restrictions on certain parameters can be formulated as equality or inequality constraints. Based on the mathematical equations, the problem can be categorized as linear, non-linear, integer, quadratic, etc. When probabilistic variables are involved, it is stochastic programming. Necessary and sufficient conditions for optimality have to be identified and the optimum solution must satisfy these conditions. The best solution among all those available is to be selected based on some criterion. The optimum solution gives a maximum performance measure as compared to other non-optimal solutions. Optimization methods can also be classified as direct and indirect. Direct methods make use of objective functions and constraint equations whereas indirect methods use properties of functions. Most of these 
approaches are suitable for programming on a computer, and hence scaling or transformation of variables can be accomplished easily.

\subsection{Classification of Optimization Algorithms}

The optimization algorithms are classified into the traditional or classical methods, evolutionary algorithms, and swarm intelligence algorithms.

Classical Methods: The classical optimization algorithms are applicable to the traditional continuous optimization functions that are differentiable. The traditional methods involve computation of the first- and second-order derivatives of the objective function in order to find maxima or minima. The solution obtained could be local or global optima but the ultimate goal of optimization is to find the global optimum. They are mostly gradient-based methods and provide deterministic solutions to optimization functions within a continuous search space. The simplex method, linear and non-linear programming, Newton's steepest descent method, Lagrangian method, integer and dynamic programming, and the Kuhn-Tucker conditions are famous classical methods, to name a few. Steepest descent is one of the classical optimization techniques for finding the minimum of a unimodal differentiable function. Figure 1.6 illustrates the trajectory of the steepest descent method. In the classical descent methods, the step size plays a major role in approaching the minimum of a unimodal function. The brute force method is a single point direct search method that searches for the optimum point starting from a single point in the search region that is bounded. The search proceeds in steps, and the step size plays a major role in convergence of the algorithm. When the number of parameters is large, the brute force method suffers from the curse of dimensionality.

The random walk method overcomes or circumvents the curse of dimensionality by searching from randomly generated points in the search space of the objective function.

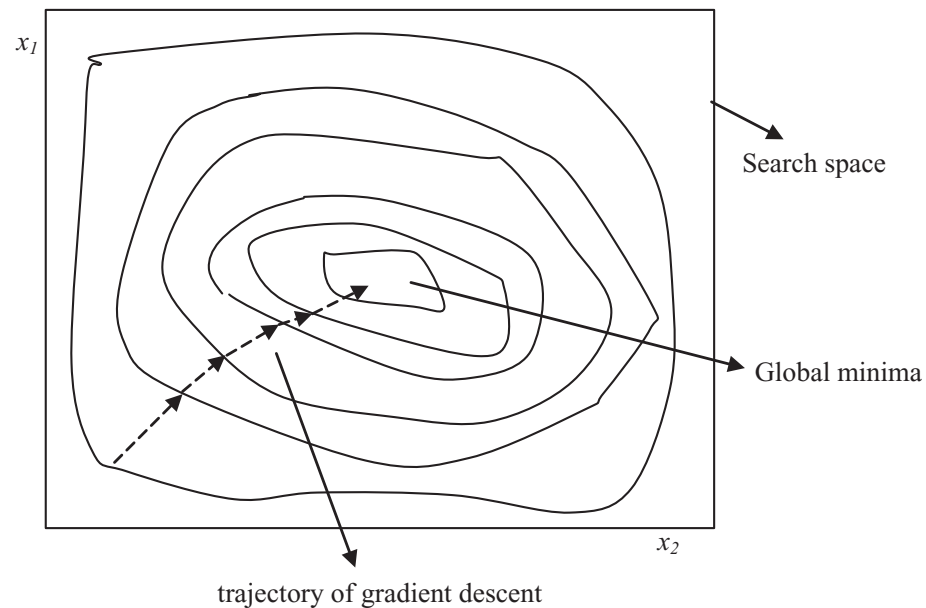

FIGURE 1.6

Illustration of gradient descent. 
The Hooke-Jeeves method is a direction or pattern search since it starts the search from a base point and has separate step size for each coordinate direction. The trial search points are compared, and if a move is found to be better, then further moves are made in the same direction. Thus the step sizes are adaptive in this method which makes it perform better than the earlier two methods. When the functions are multimodal, the starting point of the search plays a vital role in finding the global optimum since there is more than one optimum (local as well as global) point. There are other methods that are called multi-start since they search the solution space starting from multiple different initial points for each search. The search could be direct or derivative-based. Clustering could be applied where clusters of sample points are formed and one point within the cluster serves as the initial point for the search. This is limited to problems with smaller numbers of parameters since it is computationally intensive. An overview of the classical algorithms has been discussed in the following chapter.

Evolutionary Algorithms: Evolutionary algorithms are a class of optimization algorithms that are based on the biological processes of evolution. As the name implies, evolutionary computational methods are designed on the principles of evolution. They are based on the Darwinian theory of survival of the fittest and use selection, mating, reproduction, crossover, and mutation operators similar to the biological processes of evolution. Genetic algorithm (GA), genetic programming (GP), and differential evolution (DE) are three of the classical popular evolutionary algorithms that have been discussed in this book. Darwin's finches belong to a group of passerine birds that have around 15 different species within their group. They are diversified in the shape, size, and function of their beaks. Longterm study has shown evolutionary changes in their beaks. They are popularly known as Darwin's finches because Charles Darwin found and collected them from the Galapagos Islands and they formed the basis for Darwin's theory of evolution and natural selection. Figure 1.7 shows the Darwin's finches that played an important role in Darwin conceiving his theory of evolution and are a typical example of evolution.

Additionally, other evolutionary algorithms available are gene expression programming (GEP), evolutionary programming (EP), and evolutionary strategies (ES). Charles Darwin developed the theory of evolution on which these algorithms are all built upon, and they are all population-based. The fundamental goal of the theory or algorithm is survival of the species.

The transfer of a genetic program (genotype) or code from an individual to its progeny is reproduction. When there are mutations, there are differences in the transferred genetic code that are either advantageous or disadvantageous. When there are more individuals than resources, there is competition and the fitter ones tend to survive and reproduce whereas the weaker ones perish. The genotype carries genetic information from the parent to offspring with all the experience undergone by the parent so far. Phenotype is the set of characteristics or properties of the population, which is a manifestation (behavioral expression) of the genotype in a specific environment. In natural evolution, there is nonlinear mapping between genotype and phenotype and it is complex. The algorithms that are developed under the evolutionary framework are broadly referred to as evolutionary algorithms. The evolution of the population is determined by the parents, offspring, and the operators, and the algorithms differ from each other based on this combination. Let $P$ be the population size of parents, $C$ be the number of offspring or children, and after recombination the population size is $P+C$. This has to be resized or scaled down to the original size, and the operators determine the mode of resizing. The fitness of every member of the population is evaluated by the objective function, and the overall fitness is determined by the individual fitness values. 


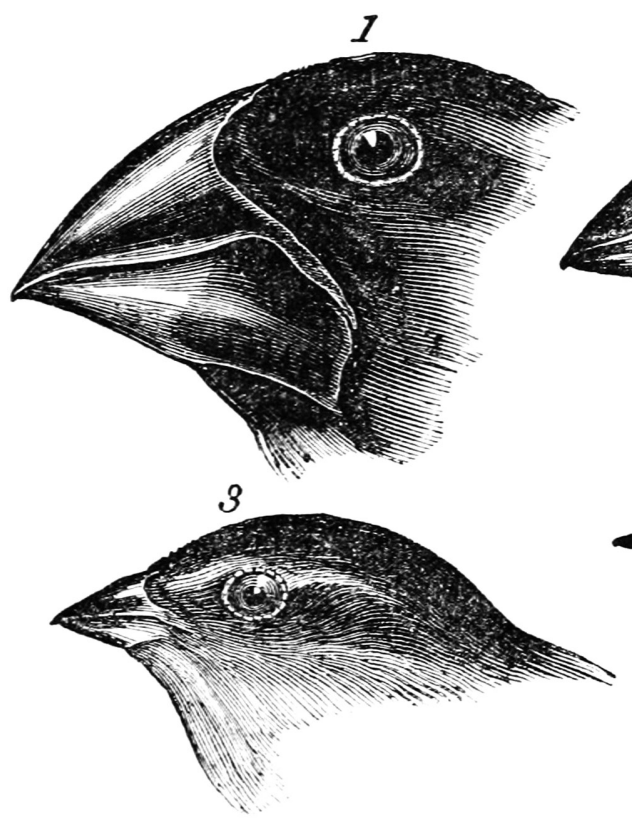

1. Geospiza magnirostris.

3. Geospiza parvula.

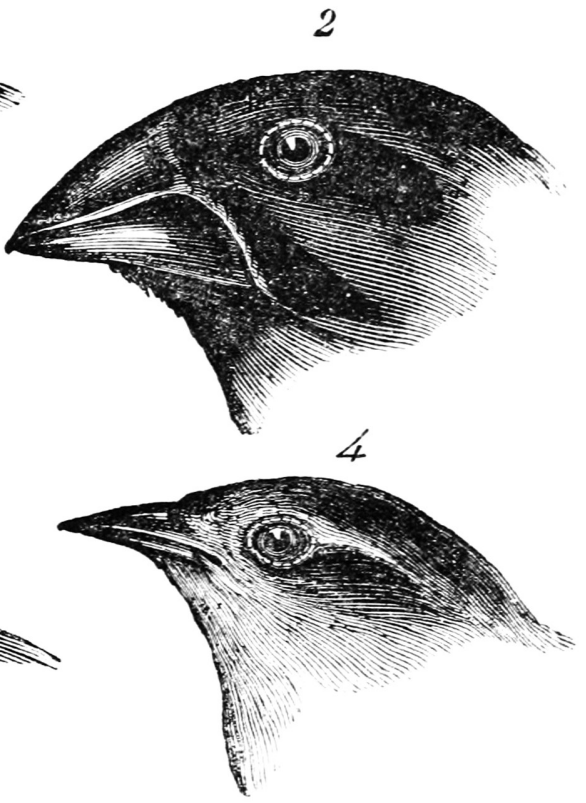

2. Geospiza fortis.

4. Certhidea olivasea.

FIGURE 1.7

Darwin's finches. [Author: John Gould (Public Domain).]

In evolutionary strategies the search is in parallel (multi-start) from multiple points in the search space. The initial population is created randomly, and parents are selected for recombination to create a population of children. The children are mutated to change their properties or characteristics; either all of them undergo mutation or it is done selectively. The next-generation population members are chosen from the total population consisting of parents and children. This resizing of the population is done based on the fitness values of the individual members.

Swarm Intelligence Algorithms: Computational intelligence (CI) is a subset of machine learning and artificial intelligence that collectively refers to algorithms with intelligence built into them. Most of the evolutionary algorithms and swarm intelligence algorithms have computational intelligence built into them. Some of the popular computational intelligence algorithms are neural networks, fuzzy logic, genetic algorithm, differential evolution, particle swarm optimization, firefly algorithm, ant colony optimization, and other swarm-based algorithms. Nature-inspired (NI) algorithms are developed based on the study of natural processes that could be physical, chemical, or biological and the behavior of animals, birds, and insects. They are mostly population-based and utilize their collective swarm intelligence in arriving at the optimum solution. Swarm intelligence (SI) algorithms have been developed based on the study of swarm behavior, and these swarms possess computational intelligence. Swarm behavior refers to the collective behavior of a group of insects, birds, fish, and animals, and swarm intelligence is the intelligence exhibited by the entire group as a whole. The individual members of the swarm follow simple rules that lead to collective and productive outcomes for the benefit of the entire swarm. They are adaptive and flexible, have good perception capabilities, and live in 


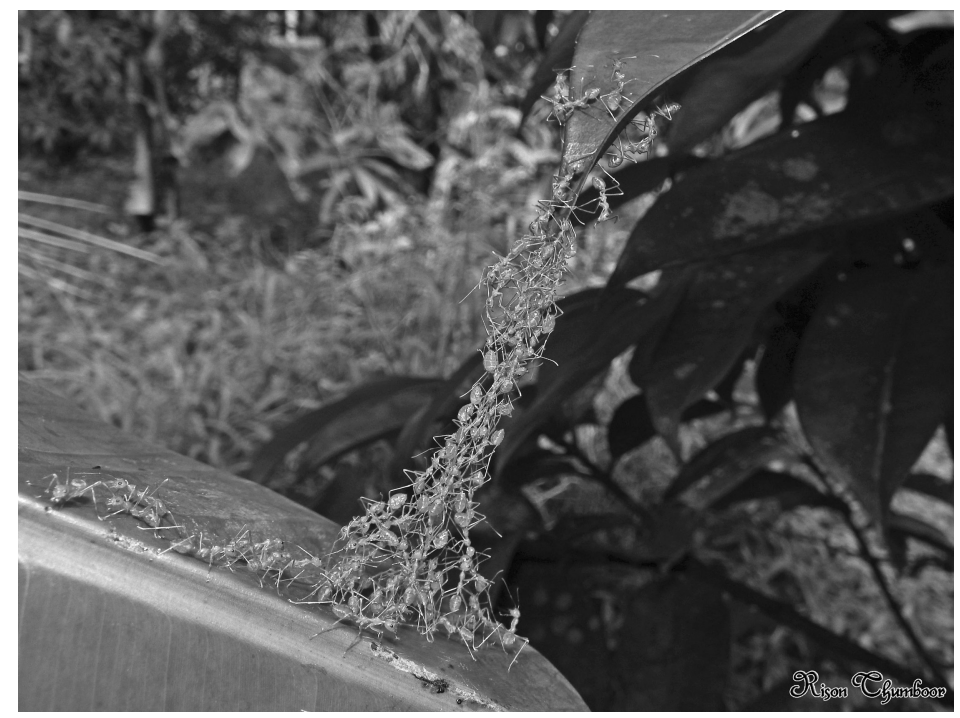

FIGURE 1.8

Weaver ants making an emergency bridge between two plants. (Author: Rose Thumboor, CC BY-SA 4.0. https:// creativecommons.org/licenses/by-sa/4.0/deed.en.)

the environment utilizing the available resources efficiently. They interact within their group as well as with the environment and behave in a self-organized manner. Most of the swarms have a hierarchy within their group and apply principles of division of labor. Their collective intelligence helps them in foraging for food, defending against predators, and societal interactions such as communication among the members of their group for the benefit of the entire swarm. Such studies have motivated the research towards development of swarm intelligence algorithms that are able to solve complex problems efficiently in finite time. Figure 1.8 shows a group of weaver ants making an emergency bridge between two plants that is a manifestation of group dynamism and collective intelligence.

Many of the classical optimization algorithms require the objective function to be a continuous function whose derivative exists. If the function is discrete in nature or if there are numerous parameters in the problem, these algorithms fail. They are not suitable for dealing with functions whose derivatives do not exist or for discrete optimization problems such as in image processing. In such cases, computational intelligence algorithms are best suited to provide the solution. They are mostly designed for searching a solution space trying to find the best solution or a close approximation. This involves some randomness in the algorithm which in itself increases the efficiency of such methods. Searching a solution space is best done using a swarm or a group of particles, and these serve as the basis for nature-inspired algorithms. They are all population-based and reduce the time of searching to find the optimum solution. SI-based techniques are mostly iterative, and they remember the past history in order to find a better solution than the previous best. Remembering the previous solutions involves memory, and many of the SI algorithms do have memory, either short-term or long-term. The algorithm stops either when a stopping criterion is attained or when the predefined maximum number of iterations is reached. The two most important components in swarm intelligence algorithms are intensification and diversification. In intensification, the search is intensified in a particular area near the 
previous best solution, whereas in diversification, the search is conducted over a wider area that was previously unexplored, looking for better solutions.

\subsection{Traveling Salesman Problem and Knapsack Problem}

Optimizing a cost function that is defined on a set of independent variables is a combinatorial optimization problem since it involves finding the right set of independent variables that maximizes or minimizes the function. The problems which generally fall into this category can be divided into two classes - those which are easy to solve and take up less time (can be solved in polynomial time) and those which take up a large amount of time and are practically infeasible to solve, called NP-hard problems. The most famous problem in computer science under the category of NP-hard is the traveling salesman problem (TSP), and other similar problems in this category are the graph partitioning (coloring) problem, job scheduling problem, and network routing problem, to name a few. These combinatorial optimization problems could be solved by the classical optimization algorithms in an infinitely long time or by the heuristic/metaheuristic optimization algorithms in finite short time that produces an optimum solution that closely approximates the actual solution.

The TSP and the knapsack problem are two typical famous examples in computer science for discrete combinatorial optimization. In the TSP, the objective function is the total distance of the Hamiltonian tour and depends on the set of cities visited and the total distance covered which is the sum of the distance of the edges connecting the cities, and in the knapsack problem the combination of the weights of the items loaded into the knapsack and their total cost determines the objective function value. Consider the traveling salesman problem which is a typical combinatorial optimization problem in computer science that has been found to be intractable or NP-hard. There are a set of cities that are interconnected. The cities are represented by nodes in a graph and the connections between the nodes are edges. Each edge has a value associated with it that could represent distance or cost. The problem is for a traveling salesman to visit each of these cities once and only once (without retracing) and go back to the starting city traveling a total minimum distance and hence incurring the minimum cost. The path in the graph traced by the traveling salesman is called the Hamiltonian tour. This is illustrated in Figure 1.9 which shows one possible Hamiltonian tour in the graph.

Consider the knapsack problem in computer science which is another typical combinatorial optimization problem. There is a knapsack or rucksack and a set of items each with a value and a weight. The items are represented by $x_{k}$ with a weight of $w_{k}$ and value $v_{k}$ where $k=1,2, \ldots, N$, assuming there are $N$ number of different items. The objective is to fill the knapsack with as many items as possible so that the total value of the items is maximum. The items can be included as a whole or in fractions, such as $c_{1} x_{1}$ where $c_{1}=1$ is one instance of the item $x_{1}, c_{1}=2$ is two instances of the item $x_{1}$, whereas $c_{1}=0.5$ is onehalf of the item $x_{1}$. The constraint is that the maximum weight of all the items put in the knapsack should not exceed its capacity of $W_{\max } \mathrm{kg}$. In this problem, the objective function is the total value of all the items in the knapsack which can be formulated as the weighted sum of the values of each item put in the knapsack. The constraint is that the sum of the weights of all the items put in the knapsack should not exceed the limit of $W_{\max } \mathrm{kg}$. This is a multi-variable, constrained optimization problem with a single objective function that is 


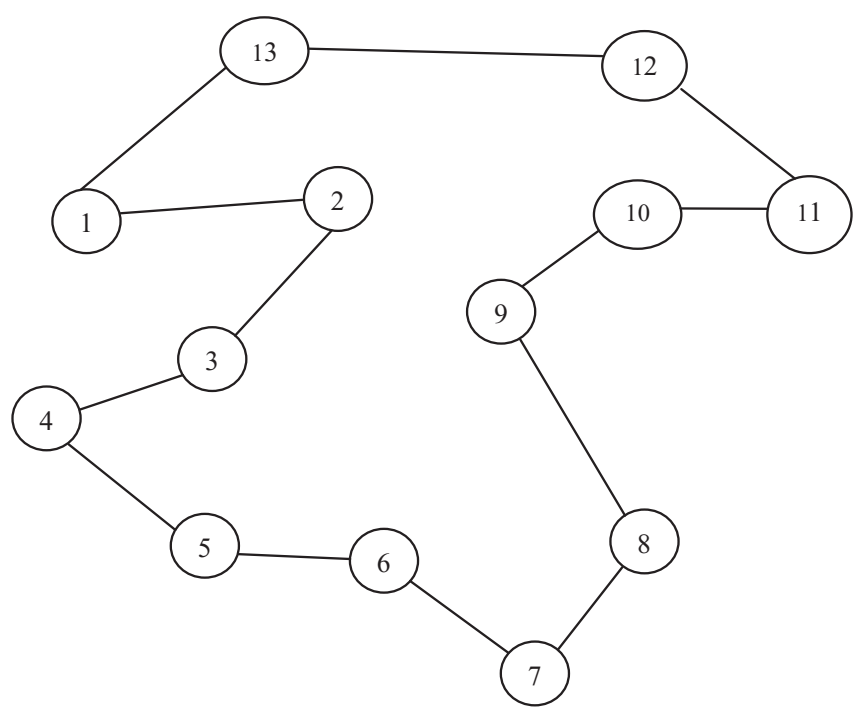

\section{FIGURE 1.9}

Hamiltonian tour for TSP.

to be maximized. This is illustrated in Figure 1.10. The objective function is a cost function that is to be maximized, given by:

$$
f(X)=c_{1} v_{1}+c_{2} v_{2}+\ldots+c_{N} v_{N}
$$

The constraint is:

$$
c_{1} w_{1}+c_{2} w_{2}+\ldots+c_{N} w_{N} \leq W_{\max }
$$

where the coefficients $c_{1}, c_{2}, \ldots, c_{N}$ are either integers or fractional numbers.

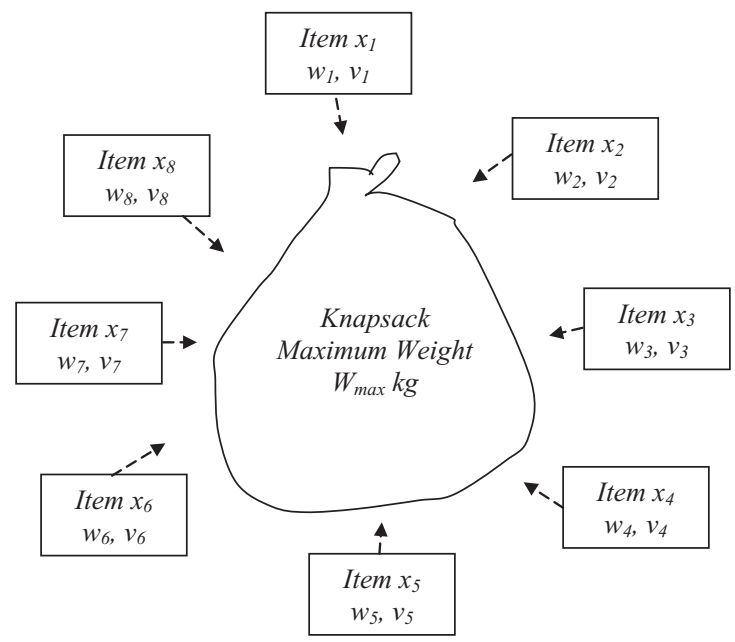

FIGURE 1.10

Knapsack problem. 


\subsection{Summary}

Any problem to be optimized should be formulated in terms of an objective function and a set of parameters. The formulation can be done either as minimization (cost) or maximization (profit) of the objective function. The algorithms proposed for such optimization problems should be able to handle linear or non-linear, continuous or discrete, differentiable or non-differentiable, unimodal or multimodal, and single- as well as multi-objective functions or search spaces. The algorithms should be able to search for the solutions in parallel (inherent parallelism) and be efficient in handling computationally intensive cost functions. The number of parameters on which the objective function or the solution depends should be as small as possible and easy to control. The algorithm should have a faster rate of convergence, and it should converge to the optimum solution in finite time.

The classical algorithms have been discussed in Chapter 2, and the nature-inspired optimization algorithms in general have been discussed in Chapter 3. Chapters 4 and 5 cover the evolutionary algorithms - genetic algorithm (GA) and genetic programming (GP). The rest of the chapters are devoted to each of the nature-inspired algorithms that are popular, with proven abilities and superiority in finding the optimum solution. The standard benchmark functions used for testing and comparing the performance of these algorithms and typical applications for evaluating these algorithms have been outlined in Chapter 19. The book concludes with a summary in Chapter 20. 


\section{References}

1. Singaresu S. Rao, Engineering Optimization, Theory and Practice, 4th edition, John Wiley \& Sons, 2009.

2. Jon C. Nash, The (Dantzig) simplex method for linear programming, IEEE Computing in Science and Engineering, Vol. 2, No. 1, pp. 29-31, January/February 2000.

3. N. Kamarkar, A new polynomial-time algorithm for linear programming, Combinatorica (Springer), Vol. 4, No. 4, pp. 373-385, December 1984.

4. C. E. Lemke, The dual method of solving the linear programming problem, Naval Research Logistics (Wiley), Vol. 1, No. 1, pp. 36-47, 1954.

5. G. B. Dantzig, L. R. Ford D. R. Fulkerson, A primal-dual algorithm for linear programs, In: Linear Inequalities and Related Systems, H. W. Kuhn and A. W. Tucker (eds) Annals of Mathematics Study, No. 38. Princeton: Princeton University Press, 1956.

6. George B Dantzig, Philip Wolfe, Decomposition principle for linear programs, Operations Research, Vol. 8, No. 1, pp. 101-111, February 1960.

7. D. R. Fulkerson, Hitchcock Transportation Problem, P-890, Santa Monica, CA: Rand Corporation, July 1956.

8. F. L. Hitchcock, The distribution of a product from several sources to numerous localities, MIT Journal of Mathematics and Physics, Vol. 20, pp. 224-230, 1941.

9. Philip Wolfe, The simplex method for quadratic programming, Econometrica, Vol. 27, No. 3, pp. 382-398, July 1959.

10. R. J. Duffin, E. L. Peterson, and Zener, C., Geometric Programming, John Wiley, New York, 1967.

11. Stephen Boyd, Seung-Jean Kim, Lieven Vandenberghe, Arash Hassibi, A tutorial on geometric programming, Optimization and Engineering (Springer), Vol. 8, pp. 67-127, March 2007.

12. E. L. Peterson, Geometric programming, In: Advances in Geometric Programming, Mathematical Concepts and Methods in Science and Engineering, Vol. 21, M. Avriel (eds). Springer, Boston, MA, pp. 31-94, 1980.

13. H. W. Kuhn and A. W. Tucker, Nonlinear Programming, Proceedings of the 2nd Berkeley Symposium on Mathematical Statistics and Probability, University of California Press, Berkeley, 1951, pp. 481-492.

14. Richard Bellman, The theory of dynamic programming, P-550, Presented to the American Mathematical Society, Wyoming, July 1954.

15. J. E. Mitchell, Branch-and-cut algorithms for integer programming, In: Encyclopedia of Optimization, C. A. Floudas and P. M. Pardalos (eds). Dordrecht, The Netherlands: Kluwer, 2001.

16. R. E. Gomory, Outline of an algorithm for integer solutions to linear programs, Bulletin of American Mathematical Society, Vol. 64, No. 5, pp. 275-278, 1958.

1. Iztok Fister Jr., Xin-She Yang, Iztok Fister, Janez Brest, Dusan Fister, A brief review of natureinspired algorithms for optimization, Elektrotehniški Vestnik, Vol. 80, No. 3, pp. 1-7, July 2013.

2. Petr Bujok, Josef Tvrdik, Radka Polakova, Comparison of nature-inspired population-based algorithms on continuous optimization problems, Swarm and Evolutionary Computation (Elsevier), Vol. 50, Article ID 100490, November 2019.

3. Xin-She Yang (Ed.), Nature inspired algorithms and applied optimization, Studies in Computational Intelligence (Springer), 2018.

4. Xin-She Yang, Nature inspired metaheuristic algorithms: Success and new challenges, Journal of Computer Engineering and Information Technology, Vol. 1, No. 1, pp. 1-3, November 2012.

5. Zhihua Cui, Rajan Alex, Rajendra Akerkar, Xin-She Yang, Recent advances on bioinspired computation, The Scientific World Journal, Hindawi Publishing Corporation, Vol. 2014, Article ID 934890, May 2014. 
6. Scott McCaulay, Biologically inspired computing algorithms: Relevance and implications for research technologies, Indiana University, Bloomington, IN. PTI Technical Report PTI-TR12-003, February 2012.

7. Xin-She Yang, Nature-Inspired Metaheuristic Algorithms, 2nd edition, Luniver Press, 2010.

8. Xin-She Yang, Nature Inspired Optimization Algorithms, 1st edition, Elsevier, London, 2014.

9. Xin-She Yang, Swarm intelligence based algorithms: A critical analysis, Evolutionary Intelligence (Springer), Vol. 7, No. 1, pp. 17-28, April 2014.

10. Xin-She Yang, Suash Deb, Simon Fong, Xingshi He, Yu-Xin Zhao, From swarm intelligence to metaheuristics: Nature inspired optimization algorithms, IEEE Computer, Vol. 49, No. 9, pp. 52-59, September 2016.

11. A. Hanif Halim, I. Ismail, Bio-inspired optimization method: A review, NNGT Journal: International Journal of Information Systems, Vol. 1, pp. 12-17, July 2014.

12. Xin-She Yang, Su Fong Chien, Tiew On Ting, Computational intelligence and metaheuristic algorithms with applications, The Scientific World Journal, Hindawi Publishing Corporation, Vol. 2014, Article ID 425853, December 2014.

13. Michael A. Lones, Metaheuristics in nature inspired algorithms, Proceedings of Genetic and Evolutionary Computation Conference (GECCO Comp '14), Vancouver, BC, Canada, pp. 1419-1422, July 2014.

14. Xin-She Yang, Suash Deb, Simon Fong, Metaheuristic algorithms: Optimal balance of intensification and diversification, Applied Mathematics and Information Sciences, An International Journal, Vol. 8, No. 3, pp. 977-983, May 2014.

15. Xin-She Yang, Suash Deb, Thomas Hanne, Xingshi He, Attraction and diffusion in natureinspired optimization algorithms, Neural Computing and Applications, Vol. 31, No. 7, pp. 19871994, July 2019.

16. D. H. Wolpert, W. G. Macready, No free lunch theorems for optimization, IEEE Transactions on Evolutionary Computation, Vol. 1, No. 1, pp. 67-82, April 1997.

17. C. Schumacher, M. D. Vose, L. D. Whitley, The no free lunch and problem description length, Proceedings of the 3rd Annual Conference on Genetic and Evolutionary Computation (GECCO '01), San Francisco, CA, United States, pp. 565-570, July 2001.

18. Giorgos Karafotias, Mark Hoogendoorn, A. E. Eiben, Parameter control in evolutionary algorithms: Trends and challenges, IEEE Transactions on Evolutionary Computation, Vol. 19, No. 2, pp. 167-187, April 2015.

1. John H. Holland, Adaptation in Natural and Artificial Systems, University of Michigan Press, Ann Arbor, MI, 1975 (re-issued by MIT Press, Cambridge, MA, 1992).

2. David E. Goldberg, Genetic Algorithms in Search, Optimization and Machine Learning, AddisonWesley, Reading, Massachussets, 1989.

3. Darrell Whitley, A genetic algorithm tutorial, Statistics and Computing, V, pp. 65-85, June 1994.

4. Melanie Mitchell, Genetic algorithms: An overview, Complexity, 1 (I), pp. 31-39, September/ October 1995.

5. John H. Holland, Genetic algorithms, Scientific American, 267 (1), pp. 66-73, July 1992.

6. Colin Reeves, Chapter 3: Genetic algorithms, In: Handbook of Metaheuristics, International Series in Operations Research and Management Science, Michel Gendreau and Jean-Yves Potvin (eds). Springer, Switzerland, pp. 109-139, 2010.

7. Abraham A., Nedjah N., Mourelle L. M. Evolutionary computation: From genetic algorithms to genetic programming, In: Nedjah N., Mourelle L.M., Abraham A. (eds) Genetic Systems Programming, Studies in Computational Intelligence (SCI), Vol. 13, pp. 1-20, Springer-Verlag, Berlin, Heidelberg, 2006.

8. Ulrich Bodenhofer, Genetic Algorithms: Theory and Applications, Lecture Notes, 3rd edition, Johannes Kepler University, Linz, October 2003.

1. John R. Koza, Hierarchical genetic algorithms operating on populations of computer programs, Proceedings of the 11th International Joint Conference on Artificial Intelligence (IJCAI '89), Vol. 1, Detroit, MI, August 1989. 
2. John R. Koza, Genetic Programming: On the Programming of Computers by Means of Natural Selection, MIT Press, Cambridge, Massachusetts, 1992.

3. Ulrich Bodenhofer, Genetic Algorithms: Theory and Applications, Lecture Notes, 3rd edition, Johannes Kepler University, Linz, October 2003.

4. Koza J.R., Poli R., Genetic programming. In: Burke E.K., Kendall G. (eds) Search Methodologies, Springer, Boston, MA, pp. 127-164, 2005.

5. Wolfgang Banzhaf, Peter Nordin, Robert E. Keller, Frank D. Francone, Genetic Programming, An Introduction, $1^{\text {st }}$ Edition, Morgan Kaufmann Publishers Inc., New York, 1998.

6. Abraham A, Nedjah N, Mourelle L M, Evolutionary computation: From genetic algorithms to genetic programming, In: Nedjah N., Mourelle L.M., Abraham A. (eds) Genetic Systems Programming, Studies in Computational Intelligence (SCI), Vol. 13, pp. 1-20, Springer-Verlag, Berlin, Heidelberg, 2006.

1. James Kennedy, Russell Eberhart, Particle swarm optimization, Proceedings of the IEEE International Conference on Neural Networks, Piscataway, NJ, Vol. IV, pp. 1942-1948. IEEE Press, 1995.

2. Mahamed G. H. Omran, Particle swarm optimization methods for pattern recognition and image processing. Ph.D. thesis. University of Pretoria, Pretoria, 2005.

3. Frans van den Bergh, An analysis of particle swarm optimizers. Ph.D. thesis. University of Pretoria, Pretoria, 2001.

4. E. O. Wilson, Sociobiology: The New Synthesis, Cambridge, MA: Belknap Press, pp. 697, 1975.

5. James Blondin, Particle swarm optimization: A tutorial, September 2009. http://cs.armstrong. edu/saad/csci8100/pso_tutorial.pdf

6. James Kennedy, Russell Eberhart, Yuhui Shi, Swarm Intelligence, Morgan Kaufmann Publishers, San Francisco, 2001.

7. Reynolds, C. W., Flocks, herds, and schools: A distributed behavioral model, Computer Graphics, Vol. 21, No. 4, SIGGRAPH '87 Conference Proceedings, pp. 25-34, 1987.

8. Millonas M. M, Swarms, Phase transitions and collective intelligence, In: Artificial Life III, C. G. Langton (ed). Reading, MA: Addison Wesley, pp. 417-443, 1993.

9. M. Clerc, J. Kennedy, The particle swarm - Explosion, stability, and convergence in a multidimensional complex space, IEEE Transactions on Evolutionary Computation, Vol. 6, No. 1, pp. 58-73, 2002.

10. Y. Shi, R. Eberhart, A modified particle swarm optimizer, 1998 IEEE International Conference on Evolutionary Computation Proceedings, IEEE World Congress on Computational Intelligence, Anchorage, AK, USA, pp. 69-73, 1998.

11. Dian Palupi Rini, Siti Mariyam Shamsuddin, Siti Sophiyati Yuhaniz, Particle swarm optimization: Technique, system and challenges, International Journal of Computer Applications, Vol. 14, No. 1, pp. 19-27, January 2011.

12. M. Clerc, The swarm and the queen: Towards a deterministic and adaptive particle swarm optimization, Proceedings of the Congress on Evolutionary Computation, Washington, DC, United States, pp. 1951-1957, July 1999.

13. Russ C. Eberhart, Y. Shi, Comparing inertia weights and constriction factors in particle swarm optimization, Proceedings of the Congress on Evolutionary Computation, San Diego, CA, United States, pp. 84-89, 2000.

1. Kenneth Price, Rainer Storn, Differential evolution - A simple and efficient adaptive scheme for global optimization over continuous spaces, Technical Report TR - 95 - 012, International Computer Science Institute, Berkeley, CA, United States, 1995.

2. Kenneth V. Price, Genetic annealing algorithm, Dr. Dobb's Journal, pp. 127 - 132, October 1994.

3. Rainer Storn, Kenneth Price, Differential evolution - A simple and efficient heuristic for global optimization over continuous spaces, Journal of Global Optimization, Vol. 11, pp. 341-359, Kluwer Academic Publishers, 1997.

4. Kenneth V. Price, Rainer M. Storn, Jouni A Lampinen, Differential Evolution - A Practical Approach to Global Optimization, Springer Natural Computing Series, Springer-Verlag, Berlin, Heidelberg, 2005. 
5. Swagatam Das, Sunkha Subhra Mullick, P. N. Suganthan, Recent advances in differential evolution - An updated survey, Swarm and Evolutionary Computation, Vol. 27, pp. 1-30, 2016.

6. Dexuan Xou, Jianhua Wu, Liqun Gao, Steven Li, A modified differential evolution algorithm for unconstrained optimization problems, Neurocomputing, Vol. 120, pp. 469-481, 2013.

7. Xiangtao Li, Minghao Yin, Modified differential evolution with self-adaptive parameters method, Journal of Combinatorial Optimization, Vol. 31, pp. 546-576, 2016.

8. Dexuan Xou, Haikuan Liu, Liqun Gao, Steven Li, A novel modified differential evolution algorithm for constrained optimization problems, Computers and Mathematics with Applications, Vol. 61, pp. 1608-1623, 2011.

1. Marco Dorigo, Gianni Di Caro, Ant colony optimization: A new meta-heuristic, Proceedings of the Congress on Evolutionary Computation CEC 1999, Vol. 2, pp. 1470-1477. IEEE, 1999.

2. Christian Blum, Ant colony optimization: Introduction and recent trends, Physics of Life Reviews, Vol. 2, pp. 353-373, 2005.

3. Marco Dorigo, Thomas Stutzle, The ant colony optimization metaheuristic: Algorithms, applications and advances, Chapter 9, In Handbook of Metaheuristics, pp. 251-285, April 2006.

4. Marco Dorigo, Mauro Birattari, Thomas Stutzle, Ant colony optimization, IEEE Computational Intelligence Magazine, Vol. 1, No. 4, pp. 28-39, November 2006.

5. Marco Dorigo, Christian Blum, Ant colony optimization theory: A survey, Theoretical Computer Science (Elsevier), Vol. 344, pp. 243-278, 2005.

6. Marco Dorigo, Thomas Stutzle, Ant Colony Optimization, MIT Press, Cambridge, MA, 2004.

7. M. Dorigo, L. M. Gambardella, Ant colonies for the traveling salesman problem, BioSystems, Vol. 43, No. 2, pp. 73-81, 1997.

8. M. Dorigo, V. Maniezzo, A. Colorni, Ant system: Optimization by a colony of cooperating agents, IEEE Transactions on Systems, Man, and Cybernetics_Part B: Cybernetics, Vol. 26, No. 1, pp. 29-41, 1996.

9. T. Stützle, H. H. Hoos, MAX-MIN ant system, Future Generation Computer Systems, Vol. 16, No. 8, pp. 889-914, 2000.

10. L. M. Gambardella, M. Dorigo, Solving symmetric and asymmetric TSPs by ant colonies, Proceedings of the 1996 IEEE International Conference on Evolutionary Computation (ICEC'96), $\mathrm{T}$. Baeck et al. (eds). Piscataway, NJ: IEEE Press, pp. 622-627, 1996.

1. D. Teodorović, Bee colony optimization (BCO). In: Innovations in Swarm Intelligence. Studies in Computational Intelligence, Vol. 248, C. P. Lim, L. C. Jain, S. Dehuri (eds). Berlin, Heidelberg: Springer, pp. 39-60, 2009.

2. Dusan Teodorovic, Panta Lucic, Goran Markovic, Mauro Dell' Orco, Bee colony optimization: Principles and applications, IEEE 8th Seminar on Neural Network Applications in Electrical Engineering, NEUREL 2006, University of Belgrade, Serbia, 25-27 September 2006.

3. Tatjana Davidovic, Dusan Teodorovic, Milica Selmic, Bee colony optimization part I: The algorithm overview, Yugoslav Journal of Operations Research, Vol. 25, No. 1, pp. 33-56, 2015.

4. Dusan Teodorovic, Milica Selmic, Tatjana Davidovic, Bee colony optimization part II: The application survey, Yugoslav Journal of Operations Research, Vol. 25, No. 2, pp. 185-219, 2015.

5. Dervis Karaboga, Bahriye Akay, A survey: Algorithms simulating bee swarm intelligence, Artificial Intelligence Review, Vol. 31, No. 1-4, pp. 61-85, 2009.

6. T. Sato, M. Hagiwara, Bee system: Finding solution by a concentrated search, Proceedings of the 1997 IEEE International Conference on Systems, Man and Cybernetics, Orlando, FL, United States, pp. 3954-3959, 12-15 October 1997.

7. Dervis Karaboga, Bahriye Basturk, A powerful and efficient algorithm for numerical function optimization: Artificial bee colony (ABC) algorithm, Journal of Global Optimization, Vol. 39, No. 3, pp. 459-471, November 2007.

8. Baris Yuce, Michael S. Packianather, Ernesto Mastrocinque, Duc Truong Pham, Alfredo Lambiase, Honey bees inspired optimization method: The bees algorithm, Insects, Vol. 4, No. 4, pp. 646-662, 2013.

9. Ebubekir Coc, The bees algorithm: Theory, improvements and applications, Ph.D. thesis. Manufacturing Engineering Centre, School of Engineering, University of Wales, UK, March 2010. 
10. D. T. Pham, A. Ghanbarzadeh, E. Koc, S. Otri, S. Rahim, M. Zaidi, The Bees Algorithm, Technical Note, Cardiff, UK: Manufacturing Engineering Center, Cardiff University, 2005.

11. H. A. Abbass, MBO: Marriage in honey bees optimization - A haplometrosis polygynous swarming approach, Proceedings of the 2001 Congress on Evolutionary Computation (IEEE), Seoul, South Korea, 27-30 May 2001.

12. C. Yang, J. Chen, X. Tu, Algorithm of fast marriage in honey bees optimization and convergence analysis, Proceedings of the IEEE International Conference on Automation and Logistics, ICAL 2007, Jinan, China, pp. 1794-1799, 18-21 August 2007.

13. A. Afshar, O. Bozorg Haddad, M. A. Marino, B. J. Adams, Honey-bee mating optimization (HBMO) algorithm for optimal reservoir operation, Journal of the Franklin Institute, Vol. 344, No. 5, pp. 452-462, 2007.

14. Petar Curkovic, Bojan Jerbic, Honey-bees optimization algorithm applied to path planning problem, International Journal of Simulation Modeling, Vol. 6, No. 3, pp. 154-164, 2007.

15. Amador-Angulo L, Castillo O, A fuzzy bee colony optimization algorithm using an interval type-2 fuzzy logic system for trajectory control of a mobile robot, In: Advances in Artificial Intelligence and Soft Computing MICAI 2015, G. Sidorov, S. Galicia-Haro (eds), Lecture Notes in Computer Science, Vol. 9413. Cham: Springer, 2015.

16. Ali-Asghar Gholami, Ramin Ayanzadeh, Elaheh Raisi, Fuzzy honey bees foraging optimization: Swarm intelligence approach for clustering, Journal of Artificial Intelligence, Vol. 7, pp. 13-23, 2014.

1. D. S. Pavlov, O. S. Kasumyan, Patterns and mechanisms of schooling behavior in fish: A review, Journal of Ichthyology, Vol. 40, Suppl. 2, pp. S163-S231, 2000.

2. Carmelo J. A. Bastos Filho, Fernando B. de Lima Neto, Anthony J. C. C. Lins, Antônio I. S. Nascimento, Marília P. Lima, A novel search algorithm based on fish school, behavior, 2008 IEEE International Conference on Systems, Man and Cybernetics (SMC 2008), pp. 2646-2651.

3. Hsing-Chih Tsai, Yong-Huang Lin, Modification of the fish swarm algorithm with particle swarm optimization formulation and communication behavior, Applied Soft Computing, Vol. 11, No. 8, pp. 5367-5374, 2011.

4. Yang Wang, Wei Zhang, Hongxing Li, Application of artificial fish swarm algorithm in image registration, Computer Modeling and New Technologies, Vol. 18, No. 12B, pp. 510-516, 2014.

5. Fran Sergio Lobato, Valder Steffen Jr., Fish swarm optimization algorithm applied to engineering system design, Latin American Journal of Solids and Structures, Vol. 11, No. 1, pp. 143-156, January 2014.

6. J. B. Monteiro-Filho, I. M. C. Albuquerque, F. B. Lima Neto, Fish school search algorithm for constrained optimization, Neural and Evolutionary Computing, pp. 1-12, November 2018.

7. Carmelo J. A. Bastos-Filho, Rodrigo P. Monteiro, Luiz F. V. Vercosa, Improving the performance of the fish school search algorithm, International Journal of Swarm Intelligence Research, Vol. 9, No. 4, pp. 21-46, October 2018.

8. Carmelo J. A. Bastos-Filho, Augusto C. S. Guimarães, Multiobjective fish school search, International Journal of Swarm Intelligence Research, Vol. 6, No. 1, pp. 23-40, 2015.

9. C. J. A. Bastos-Filho, D. O. Nascimento, An enhanced fish school search algorithm, Proceedings of the 2013 BRICS Congress on Computational Intelligence and 11th Brazilian Congress on Computational Intelligence, Washington, DC, United Sattes, pp. 152-157, 8-11 Septembr 2013.

1. X.-S. Yang, S. Deb, Engineering optimisation by cuckoo search, International Journal of Mathematical Modelling and Numerical Optimisation, Vol. 1, No. 4, pp. 330-343, 2010.

2. X.-S. Yang, S. Deb, Cuckoo search via Lévy flights, In: Proceedings of World Congress on Nature and Biologically Inspired Computing (NaBIC 2009)Figu, Coimbatore, India, pp. 210-214, December 2009, published by IEEE, USA, ISBN: 978-1-4244-5053-4.

3. Iztok Fister Jr., Dusan Fister, Iztok Fister, A comprehensive review of cuckoo search: Variants and hybrids, International Journal of Mathematical Modelling and Numerical Optimisation, Vol. 4, No. 4, pp. 387-409, 2013.

4. P. Barthelemy, J. Bertolotti, D. S. Wiersma, A Lévy flight for light, Nature, Vol. 453, pp. 495-498, 2008. 
5. I. Fister Jr., X. S. Yang, D. Fister, I. Fister, Cuckoo search: A brief literature review, In: Cuckoo Search and Firefly Algorithm: Theory and Applications, Studies in Computational Intelligence, Vol. 516, pp. 49-62, 2014.

6. Aziz Quaarab, Belaid Ahiod, Xin-She Yang, Discrete cuckoo search algorithm for the traveling salesman problem, Neural Computing and Applications, Vol. 24, No. 7-8, pp. 1659-1669, June 2014.

7. L. A. M. Pereira, D. Rodrigues, T. N. S. Almeida, C. C. O. Ramos, A. N. Souza, X.-S. Yang, J. P. Papa, A binary cuckoo search and its application for feature selection, 2013 IEEE International Symposium on Circuits and Systems (ISCAS2013).

8. Waleed Yamany, Nashwa El-Bendary, Aboul Ella Hassanien, Eid Emary, Multi-objective cuckoo search optimization for dimensionality reduction, 20th International Conference on Knowledge Based and Intelligent Information and Engineering Systems, Procedia Computer Science (Elsevier), Vol. 96, pp. 207-215, 2016.

9. X.-S. Yang, S. Deb, Multiobjective cuckoo search for design optimization, Computers and Operations Research, Vol. 40, pp. 1616-1624, 2013.

1. X.-S. Yang, Engineering Optimization: An Introduction with Metaheuristic Applications, Chichester: Wiley, 2010.

2. X.-S. Yang, Firefly algorithm, Lévy flights and global optimization, In: Research and Development in Intelligent Systems, XXVI, M. Bramer, R. Ellis, M. Petridis (eds). London: Springer, pp. 209218, 2010.

3. J. Kwiecien, B. Filipowicz, Firefly algorithm in optimization of queueing systems, Bulletin of the Polish Academy of Sciences, Technical Sciences, Vol. 60, No. 2, 2012.

4. Ming-Huwi Horng, Vector quantization using the firefly algorithm for image compression, Expert Systems with Applications, Vol. 39, pp. 1078-1091, 2012.

5. R. B. Francisco, M. F. P. Costa, A. M. A. C. Rocha, Experiments with firefly algorithm, In: Computational Science and Its Applications, ICCSA 2014, Lecture Notes in Computer Science, Vol. 8580, B. Murgante et al. (eds). Cham: Springer, 2014.

6. X.-S. Yang, Xingshe He, Firefly algorithm: Recent advances and applications, International Journal of Swarm Intelligence, Vol. 1, No. 1, pp. 36-50, 2013.

7. X.-S. Yang, Firefly Algorithms for Multimodal Optimization, LNCS 5792, pp. 169-178, Berlin, Heidelberg: Springer-Verlag, 2009.

1. X.-S. Yang, A new metaheuristic bat-inspired algorithm, In: Nature Inspired Cooperative Strategies for Optimization (NISCO 2010), J. R. Gonzalez et al. (ed), Studies in Computational Intelligence, 284. Berlin: Springer, pp. 65-74, 2010.

2. X.-S. Yang, Bat algorithm for multi-objective optimization, International Journal of Bio-Inspired Computation, Vol. 3, No. 5, pp. 267-274, 2011.

3. Selim Yilmaz, Ecir U. Kucuksille, Improved bat algorithm on continuous optimization problems, Lecture Notes on Software Engineering, Vol. 1, No. 3, pp. 279-283, August 2013.

4. Chiranjeevi Karri, Umaranjan Jena, Fast vector quantization using a bat algorithm for image compression, Engineering Science and Technology: An International Journal, Vol. 19, pp. 769-781, 2016.

5. Eneko Osaba, Xin-She Yang, Fernando Diaz, Pedro Lopez-Garcia, Roberto Carballedo, An improved discrete bat algorithm for symmetric and asymmetric traveling salesman problems, Engineering Applications of Artificial Intelligence, Vol. 48, pp. 59-71, 2016.

6. X.-S. Yang, Bat algorithm: Literature review and applications, International Journal of BioInspired Computation, Vol. 5, No. 3, pp. 141-149, 2013.

7. J. Senthilnath, Sushant Kulkarni, J. A. Benediktsson, X.-S. Yang, A novel approach for multispectral satellite image classification based on the bat algorithm, IEEE Geoscience and Remote Sensing Letters, Vol. 13, No. 4, pp. 599-603, 2016.

8. X.-S. Yang, Mehmet Karamanoglu, Simon Fong, Bat algorithm for topology optimization in microelectronic applications, IEEE First International Conference on Future Generation Technologies, pp. 150-155, 2012. 
9. Adis Alihodzic, Milan Tuba, Bat algorithm for image thresholding, Recent Researches in Telecommunications, Informatics, Electronics and Signal Processing, IEEE 12th International Conference on Signal Processing, pp. 364-369, 2013.

10. Jian Xie, Yongquan Zhou, Huan Chen, A novel bat algorithm based on differential operator and Levy flights trajectory, Computational Intelligence and Neuroscience, Vol. 2013, pp. 1-13, 2013.

1. Xin-She Yang, Flower pollination algorithm for global optimization, In: Unconventional Computation and Natural Computation 2012, Lecture Notes in Computer Science, Vol. 7445, pp. 240 249, 2012.

2. L. Chittka, J. Thomson, Nickolas M. Waser, Flower constancy, insect psychology and plant evolution, Naturwissenschaften, Vol. 86, Springer-Verlag, pp. 361-377, 1999.

3. Xin-She Yang, Mehmet Karamanoglu, Xingshi He, Multi-objective flower algorithm for optimization, Procedia Computer Science, Vol. 18, pp. 861-868, 2013.

4. Rui Wang, Yongquan Zhou, Shilei Qiao, Kang Huang, Flower pollination algorithm with bee pollinator for cluster analysis, Information Processing Letters, Vol. 116, pp. 1-14, 2016.

5. A. Emad Nabil, Modified flower pollination algorithm for global optimization, Expert Systems with Applications, Vol. 57, pp. 192-203, 2016.

6. F. R. Fekety, The clonal selection theory of acquired immunity, Yale Journal of Biology and Medicine, Vol. 32, p. 480, 1960.

7. O. Abdel Raouf, M. Abdel-Baset, I. El-henawy, A new hybrid flower pollination algorithm for solving constrained global optimization problems, International Journal of Applied Operational Research, Vol. 4, No. 2, pp. 1-13, Spring 2014.

8. Szymon Lukasik, Piotr A. Kowalski, Study of flower pollination algorithm for continuous optimization, In: Intelligent Systems, Editors: P. Angelov, K. T. Atanassov, L. Doukovska, M. Hadijski, V. Jotsov, J. Kacprzyk, N. Kasabov, S. Sotirov, E. Szmidt, S. Zadrozny, Springer, pp. 451-459, 2015.

9. Rohit Salgotra, Urvinder Singh, Application of mutation operators to flower pollination algorithm, Expert Systems with Applications, Vol. 79, pp. 112-129, 2017.

10. Nazmus Sakib, Md. Wasi Ul Kabir, Md. Subbir Rahman, Mohammad Shafiul Alam, A comparative study of flower pollination algorithm and bat algorithm on continuous optimization problems, International Journal of Applied Information Systems, Vol. 7, No. 9, September 2014.

11. Marwa Sharawi, E. Emary, Imane Aly Saroit, Hesham El Mahdy, Flower pollination optimization algorithm for wireless sensor network lifetime global optimization, International Journal of Soft Computing and Engineering, Vol. 5, No. 3, pp. 54-59, July 2014.

12. P. Dinakara Prasad Reddy, V. C. Veera Reddy, T. Gowri Manohar, Application of flower pollination algorithm for optimal placement and sizing of distributed generation in distribution systems, Journal of Electrical Systems and Information Technology, Vol. 3, pp. 14-22, 2016.

1. E. Emary, Hossam M. Zawbaa, Aboul Ella Hassanien, Binary grey wolf optimization approaches for feature selection, Neurocomputing, Vol. 172, pp. 371-381, January 2016.

2. Linguo Li, Lijuan Sun, Jin Qi, Bin Xu, Shujing Li, Modified discrete grey wolf optimizer algorithm for multilevel image thresholding, Computational Intelligence and Neuroscience (Hindawi), Vol. 2017, pp. 1-17, 2017.

3. Seyedali Mirjalili, Shahrzad Saremi, Seyed Mohammad Mirjalili, Leandrodos S Coelho, Multiobjective grey wolf optimizer: A novel algorithm for multi-criterion optimization, Expert Systems with Applications (Elsevier) Vol. 47, pp. 106-119, April 2016.

4. E. Emary, Waleed Yamany, Aboul Ella Hassanien, Vaclav Snasel, Multi-objective gray-wolf optimization for attribute reduction, International Conference on Communication, Management and Information Technology (ICCMIT 2015), Procedia Computer Science, Vol. 65, pp. 623-632, 2015.

5. Nitin Mittal, Urvinder Singh, Balwinder Singh Sohi, Modified grey wolf optimizer for global engineering optimization, Applied Computational Intelligence and Soft Computing (Hindawi), Vol. 2016, pp. 1-17, 2016.

6. R. Rajakumar, J. Amudhavel, P. Dhavachelvan, T. Vengattaraman, GWO-LPWSN: Grey wolf optimization algorithm for node localization problem in wireless sensor networks, Journal of Computer Networks and Communications (Hindawi), Vol. 2017, pp. 1-11, 2017. 
7. E. Emary, Hossam M. Zawbaa, Crina Grosan, Experienced gray wolf optimization through reinforcement learning and neural networks, IEEE Transactions on Neural Networks and Learning Systems, Vol. 29, No. 3, pp. 681-694, March 2018.

8. Duangjai Jitkongchuen, Pongsak Phaidang, Piyalak Pongtawevirat, Grey wolf optimization algorithm with invasion-based migration operation, 2016 IEEE /ACIS 15th International Conference on Computer and Information Science (ICIS), Japan.

9. Mehak Kohli, Sankalp Arora, Chaotic grey wolf optimization algorithm for constrained optimization problems, Journal of Computational Design and Engineering, Vol. 5, No. 4, pp. 458-472, October 2018.

10. Radu-Emil Precup, Radu-Codrut David, Alexandra-lulia Szedlak-Stinan, Emil M. Petriu, Florin Dragan, An easily understandable grey wolf optimizer and its application to fuzzy controller tuning, Algorithms, Vol. 10, No. 2:68, pp. 1-15, 2017.

11. Qiang Li, Huiling Chen, Hui Huang, Xuehua Zhao, ZhenNao Cai, Changfei Tong, Wenbin Liu, Xin Tian, An enhanced grey wolf optimization based feature selection wrapped kernel extreme learning machine for medical diagnosis, Computational and Mathematical Models in Medicine (Hindawi), Vol. 2017, pp. 1-15, 2017.

12. Hui Xu, Xiang Liu, Jun Su, An improved grey wolf optimizer algorithm integrated with cuckoo search, 9th IEEE International Conference on Intelligent Data Acquisition and Advanced Computing Systems: Technology and Applications (IDAACS), Bucharest, Romania, September 2017.

1. G.-G. Wang, S. Deb, X.-Z. Gao L. dos Santos Coelho, Elephant herding optimization, IEEE 3rd International Symposium on Computational and Business Intelligence (ISCBI), Bali, Indonesia, 2015.

2. G.-G. Wang, S. Deb, X.-Z. Gao, L. dos Santos Coelho, A new metaheuristic optimization algorithm motivated by elephant herding behaviour, International Journal of Bio-Inspired Computation, Vol. 8, No. 6, pp. 394-409, 2016.

3. Alaa A. K. Ismaeel, Islam A. Elshaarawy, Essam H. Houssein, Fatma Helmy Ismail, Aboul Ella Hassanien, Enhanced elephant herding optimization for global optimization, IEEE Access, Vol. 7, pp. 34738-34752, March 2019.

4. Mostafa A. Elhosseini, Ragab A. El Sahiemy, Yasser I. Rashwan, X. Z. Gao, On the performance improvement of elephant herding optimization algorithm, Knowledge Based Systems (Elsevier), Vol. 166, pp. 58-70, February 2019.

5. Viktor Tuba, Marko Beko, Milan Tuba, Performance of elephant herding optimization algorithm on CEC2013 real parameter single objective optimization, WSEAS Transactions on Systems, Vol. 16, pp. 100-105, 2017.

1. Alireza Askarzadeh, A novel metaheuristic method for solving constrained engineering optimization problems: Crow search algorithm, Computers and Structures (Elsevier), Vol. 169, pp. $1-12,2016$.

2. Seyed Hamid Reza Pasandideh, Soheyl Khalilpurazari, Sine cosine crow search algorithm: A powerful hybrid meta heuristic for global optimization, Third International Conference on Artificial Intelligence and Soft Computing, August 2017.

3. Alireza Balavand, Ali Husseinzadeh Kashan, Abbas Saghaei, Automatic clustering based on crow search algorithm-Kmeans (CSA-Kmeans) and data envelopment analysis (DEA), International Journal of Computational Intelligence Systems, Vol. 11, No. 1, pp. 1322-1337, 2018.

4. Almoataz Y. Abdelaziz, Ahmed Fathy, A novel approach based on crow search algorithm for optimal selection of conductor size in radial distribution networks, Engineering Science and Technology, an International Journal (Elsevier), Vol. 20, pp. 391-402, 2017.

5. Primitivo Diaz, Marco Perez-Cisneros, Erik Cuevas, Omar Avelos, Jorge Galvez, Salvador Hinojosa, Daniel Zaldivar, An improved crow search algorithm applied to energy problems, energies, Vol. 11, No. 3, pp. 571, 2018.

6. Rizk M. Rizk-Allah, Aboul Ella Hassanien, Siddhartha Bhattacharyya, Chaotic crow search algorithm for fractional optimization problems, Applied Soft Computing (Elsevier), Vol. 71, pp. 1161-1175, 2018. 
7. Antono Adhi, Budi Santosa, Nurhadi Siswanto, A meta-heuristic method for solving scheduling problem: Crow search algorithm, International Conference on Industrial and System Engineering (IConISE), 2017.

8. Rachid Habachi, Achraf Touil, Abdelkabir Charkaoui, Abdelwahed Echchatbi, Eagle strategy based crow search algorithm for solving unit commitment problem in smart grid system, Indonesian Journal of Electrical Engineering and Computer Science, Vol. 12, No. 1, pp. 17-29, October 2018.

1. Anthony Brabazon, Wei Cui, Michael O'Neill, The raven roosting optimisation algorithm, Soft Computing (Springer-Verlag), Vol. 20, pp. 525-545, 2016.

2. J. Wright, R. Stone, N. Brown, Communal roosts as structured information centres in the raven, Corvus Corax, Journal of Animal Ecology, Vol. 72, No. 6, pp. 1003-1014, 2003.

3. Richard B. Stiehl, Observations of a large roost of common ravens, The Cooper Ornithological Society, Condor, Vol. 83, pp. 78, 1981.

4. John M. Marzluff, Bernd Heinrich, Raven roosts are still information centres, Animal Behaviour, Vol. 61, pp. F14-F15, 2001.

5. Shadi Torabi, Faramarz Safi-Esfahani, Improved raven roosting optimization algorithm, Swarm and Evolutionary Computation (Elsevier), Vol. 40, pp. 144-154, 2018. 


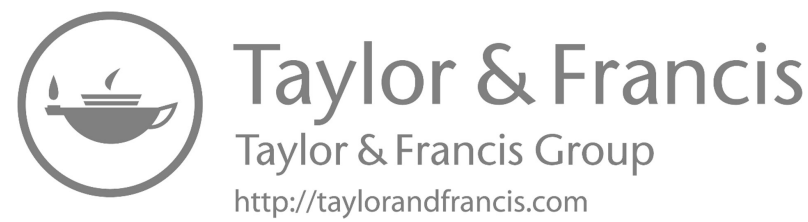

